\title{
COSMIC EVOLUTION OF RADIO SELECTED ACTIVE GALACTIC NUCLEI IN THE COSMOS FIELD*
}

\author{
V. Smolčić ${ }^{1}$, G. Zamorani ${ }^{2}$, E. Schinnerer ${ }^{3}$, S. Bardelli ${ }^{2}$, M. Bondi ${ }^{4}$, L. Bîrzan ${ }^{5,6}$, C. L. Carilli ${ }^{7}$, P. Ciliegi ${ }^{2}$, \\ M. Elvis ${ }^{8}$, C. D. Impey ${ }^{9}$, A. M. Koekemoer ${ }^{10}$, A. Merloni ${ }^{11,12}$, T. Paglione ${ }^{13,14}$, M. Salvato ${ }^{1}$, M. Scodeggio ${ }^{15}$, \\ N. SCOVILLE ${ }^{1}$, AND J. R. TRUMP ${ }^{9}$ \\ 1 California Institute of Technology, MC 105-24, 1200 East California Boulevard, Pasadena, CA 91125, USA \\ 2 INAF-Osservatorio Astronomico di Bologna, via Ranzani 1, 40127, Bologna, Italy \\ 3 Max Planck Institut für Astronomie, Königstuhl 17, Heidelberg, D-69117, Germany \\ ${ }^{4}$ INAF-Istituto di Radioastronomia, via Gobetti 101, 40129 Bologna, Italy \\ 5 Department of Astronomy and Astrophysics, Pennsylvania State University, University Park, PA 16802, USA \\ ${ }^{6}$ Department of Physics and Astronomy, Ohio University, Clippinger Laboratories, Athens, OH 45701, USA \\ ${ }^{7}$ National Radio Astronomy Observatory, P.O. Box 0, Socorro, NM 87801-0387, USA \\ ${ }^{8}$ Harvard-Smithsonian Center for Astrophysics, Cambridge, MA 02138, USA \\ ${ }^{9}$ Steward Observatory, University of Arizona, Tucson, AZ 85721, USA \\ ${ }^{10}$ Space Telescope Science Institute, 3700 San Martin Drive, Baltimore, MD 21218, USA \\ ${ }^{11}$ Excellence Cluster Universe, Technische Universität München Boltzmannstr. 2, D-85748, Garching, Germany \\ 12 Max-Planck-Institut für Extraterrestrische Physik, Giessenbachstr., D-85741 Garching, Germany \\ 13 York College, City University of New York, 94-20 Guy R. Brewer Boulevard, Jamaica, NY 11451, USA \\ ${ }^{14}$ American Museum of Natural History, Central Park West at 79th Street, New York, NY 10024, USA \\ 15 IASF Milano-INAF, Via Bassini 15, I-20133, Milan, Italy \\ Received 2008 September 25; accepted 2009 January 21; published 2009 April 13
}

\begin{abstract}
We explore the cosmic evolution of radio luminous active galactic nuclei (AGNs) with low radio powers $\left(L_{1.4 \mathrm{GHz}} \lesssim 5 \times 10^{25} \mathrm{~W} \mathrm{~Hz}{ }^{-1}\right)$ out to $z=1.3$ using to date the largest sample of $\sim 600$ low-luminosity radio AGN at intermediate redshift drawn from the VLA-COSMOS survey. We derive the radio-luminosity function for these AGNs, and its evolution with cosmic time assuming two extreme cases: (1) pure luminosity and (2) pure density evolution. The former and latter yield $L_{*} \propto(1+z)^{0.8 \pm 0.1}$, and $\Phi_{*} \propto(1+z)^{1.1 \pm 0.1}$, respectively, both implying a fairly modest change in properties of low-radio-power AGNs since $z=1.3$. We show that this is in stark contrast with the evolution of powerful $\left(L_{1.4 \mathrm{GHz}}>5 \times 10^{25} \mathrm{~W} \mathrm{~Hz}^{-1}\right)$ radio AGN over the same cosmic time interval, constrained using the 3CRR, 6CE, and 7CRS radio surveys by Willot et al. We demonstrate that this can be explained through differences in black hole fueling and triggering mechanisms, and a dichotomy in host galaxy properties of weak and powerful AGNs. Our findings suggest that high- and low-radio-power AGN activities are triggered in different stages during the formation of massive red galaxies. We show that weak radio AGN occur in the most massive galaxies already at $z \sim 1$, and they may significantly contribute to the heating of their surrounding medium and thus inhibit gas accretion onto their host galaxies, as recently suggested for the "radio mode" in cosmological models.
\end{abstract}

Key words: cosmology: observations - galaxies: active - galaxies: evolution - galaxies: fundamental parameters radio continuum: galaxies

Online-only material: color figures

\section{INTRODUCTION}

\subsection{AGN Feedback: The Impact of Radio Sources on Galaxy Formation}

Radio emission from active galactic nuclei (AGNs) has recently been invoked in cosmological models as a significant ingredient in the process of galaxy formation ("AGN feedback"). Given that, in the past, cosmological models led to a systematic over-prediction of the high-mass end of the galaxy stellar mass function (e.g., White \& Rees 1978; White \& Frenk 1991), the implementation of gas heating through energetic radio outflows - the "radio" mode ${ }^{16}$ - managed to reproduce well many observed galaxy properties (e.g., the galaxies' stellar mass function, color bimodality; Croton et al. 2006; Bower et al. 2006). The particular choice of a "radio mode" as the relevant heating mechanism has been motivated by many observational results verifying the interplay between the emission of radio

\footnotetext{
* Based on observations with the National Radio Astronomy Observatory which is a facility of the National Science Foundation operated under cooperative agreement by Associated Universities, Inc.

16 The "radio mode" is defined here as given in Croton et al. (2006).
}

galaxies in galaxy clusters, and the cluster's hot X-ray emitting gas on large scales (e.g., Fabian et al. 2003; Forman et al. 2005).

In the centers of galaxy clusters the radiative cooling time of the intracluster medium (ICM) is shorter than the age of the cluster. Thus, the central ICM is expected to be significantly colder compared to outskirt regions (such clusters are referred to as "cooling flow clusters"). However, generally the expected cool X-ray phases are not observed, and this is known as the "cooling flow problem". The most likely solution to this problem is thought to be radio galaxies as it is usually observed that the synchrotron plasma ejected by their radio jets inflates bubbles in the hot X-ray emitting cluster gas and thereby heats it (see Fabian 1994 for a review). In theoretical models, a similar interplay on smaller scales has been assumed to be at work between the radio outflows of a galaxy and its surrounding hot-gas halo.

The first observational evidence for the "radio mode" feedback in the context of galaxy formation has been found by Best et al. (2006). Based on X-ray and radio observations of a local sample of massive elliptical galaxies (Best et al. 2005) they have shown that the radio-source heating may indeed balance the radiative energy losses from the hot gas surrounding the galaxy, as postulated in the models. Based on an independent sample of radio galaxies in galaxy clusters (studied by Bîrzan et al. 2004) 
Best et al. (2006) have correlated the mechanical heating provided by the radio sources on their surrounding medium with monochromatic $20 \mathrm{~cm}$ radio power. Combining this with the local radio AGN luminosity function (LF; Best et al. 2005), they have found that the volume-averaged mechanical energy heating rate of local radio-luminous AGN is about a factor of 10-20 lower than predicted by the Croton et al. (2006) model. They assigned this difference to a systematic overprediction of the heating rate in the model (by a factor of 2-3) and the intention of the model to jointly describe both the large- and small-scale heating from radio sources (i.e., on both cluster and galaxy scales). More recent calculations of the volume-averaged mechanical energy heating rate due to AGN (Körding et al. 2008; Merloni \& Heinz 2008) yield higher values at $z=0$ compared to the results of Best et al. (2006), and thus closer to the Croton et al. (2006) prediction.

To date no clear picture exists on how the AGN radio mode feedback works. More observations are needed to provide better constraints on the physics of this process, as well as its evolution with cosmic time. Here we attempt to shed light on the latter utilizing a large statistical sample of radio AGN, drawn from the VLA-COSMOS ${ }^{17}$ survey (Schinnerer et al. 2007).

\subsection{Radio-Luminous AGN and their Evolution}

Fanaroff \& Riley (1974, FR hereafter) were the first to note that the radio-luminous AGN population consists of two apparently distinct types of sources, referred to as FR types I and II, with prominent differences in both radio morphology and luminosity. The radio emission from FR I radio galaxies is core dominated, while FR IIs are edge-brightened with highly collimated large-scale jets. FR IIs are typically more powerful in the radio than FR Is, and a dividing luminosity of $L_{178 \mathrm{MHz}} \sim 2.5 \times 10^{26} \mathrm{~W} \mathrm{~Hz}^{-1}$ (corresponding to $L_{1.4 \mathrm{GHz}} \sim 6 \times$ $\left.10^{25} \mathrm{~W} \mathrm{~Hz}^{-1}\right)^{18}$ has been suggested (Fanaroff \& Riley 1974). It has been later shown (Ledlow \& Owen 1996) that the FR I/FR II division is also a function of the host galaxy optical luminosity (with a higher dividing radio luminosity for higher optical host luminosity).

An alternative way of classifying radio $A G N$ is based on the existence of high-excitation (HE) emission lines in the optical spectra of their host galaxies (Hine \& Longair 1979; Laing et al. 1994). In this scheme, objects without high-excitation emission lines are referred to as low-excitation (LE) radio galaxies, and they are most common at low radio luminosities. Almost all FR I radio galaxies are LE sources, while optical hosts of the most powerful radio sources, i.e., FR IIs, usually have strong emission lines. It is noteworthy, however, that the correspondence between the FR class and the presence of emission lines is not one-to-one. Many FR II galaxies have been found to be LE radio galaxies (e.g., Evans et al. 2006).

Recently, strong evidence (Evans et al. 2006; Hardcastle et al. 2006, 2007) has emerged supporting the idea that low- and highexcitation radio AGNs (hereafter LERAGN and HERAGN, respectively) reflect different modes of black hole (BH) accretion. Independent studies have shown that (1) LERAGN are a class of radio-luminous AGNs that accrete radiatively inefficiently

\footnotetext{
17 The VLA is operated by the National Radio Astronomy Observatory, which is a facility of the National Science Foundation, operated under cooperative agreement by Associated Universities, IIIC.

18 A spectral index of $\alpha=0.7\left(F_{v} \propto v^{-\alpha}\right.$; where $F_{v}$ is the radio flux density) is assumed throughout the paper.
}

(Evans et al. 2006; Hardcastle et al. 2006), and (2) Bondi accretion of the hot X-ray emitting intergalactic medium (IGM) is sufficient to power the jets of low-power radio galaxies in the centers of galaxy clusters (based on a sample of nearby galaxies; Allen et al. 2006). Based on these findings Hardcastle et al. (2007) have developed a model in which all LE radio sources are powered by radiatively inefficient accretion (i.e., at subEddington accretion rates) of the hot phase of the IGM, while $\mathrm{HE}$ sources are powered by radiatively efficient accretion (at $\sim$ Eddington rates) of cold gas (that is in general unrelated to the hot IGM; see also Ho 2005; Merloni \& Heinz 2008). This model successfully explains a variety of properties of radio-luminous AGN, such as their environments, signs of galaxy mergers in the hosts of powerful $(\mathrm{HE})$ radio sources, and the break of the radio AGN LF (for details see Hardcastle et al. 2007 and references therein).

In the past two decades it has become clear that radioluminous AGN evolve differentially: low-power sources evolve less strongly than high-power sources. Numerous studies of high-luminosity radio AGN $\left(L_{2.7 \mathrm{GHz}} \gtrsim 10^{25} \mathrm{~W} \mathrm{~Hz}^{-1} \mathrm{sr}^{-1}\right.$ corresponding to $L_{1.4 \mathrm{GHz}} \sim 2 \times 10^{26} \mathrm{~W} \mathrm{~Hz}{ }^{-1}$; Dunlop \& Peacock 1990; Willott et al. 2001) have found a strong positive density evolution with redshift of these sources out to a redshift peak of $z \sim 2$. Beyond this redshift their comoving volume density starts declining (a possible sharp density cut-off has been suggested by Dunlop \& Peacock 1990, but has not been confirmed by Willott et al. 2001). Such a decline would be consistent with the results obtained via optical surveys (Schmidt et al. 1995) and, recently, X-ray surveys (Silverman et al. 2008; Brusa et al. 2009).

Analyzing the evolution of lower-power radio AGN $\left(L_{1.4 \mathrm{GHz}}>2 \times 10^{25} \mathrm{~W} \mathrm{~Hz}^{-1}\right)$ Waddington et al. (2001) have found a significantly slower evolution of this population, with the comoving volume density turnover occurring at a lower redshift $(z \sim 1-1.5)$, compared to the high-power radio population. However different radio-optical surveys, probing radio luminosities of $\sim 10^{25} \mathrm{~W} \mathrm{~Hz}^{-1}$ are still somewhat controversial. While Clewley \& Jarvis (2004) and Sadler et al. (2007) found no evidence for particularly strong evolution out to $z \sim 0.7$, Cowie et al. (2004) have found a strong density evolution out to $z \sim 1.5$. Thus, although on average a weaker evolution of lowpower (compared to high-power) radio AGN is implied, it is still not very well understood how the low-luminosity radio AGN evolve with redshift. In this work we use the VLA-COSMOS AGN sample of low-luminosity $\left(96 \%\right.$ have $L_{1.4 \mathrm{GHz}}<10^{25} \mathrm{~W}$ $\mathrm{Hz}^{-1}$ ) radio sources in order to comprehensively constrain the evolution of the low-power radio AGN out to $z=1.3$. We combine our results with the new findings and ideas on the cosmological relevance of radio AGN in order to study the impact of radio-luminous AGN on galaxy formation.

The paper is outlined as follows. In Section 2 we define the VLA-COSMOS AGN sample. In Section 3, we derive the radioLF for the VLA-COSMOS AGN, and extend it to high radio powers using the results from Willott et al. (2001). In Section 4, we constrain the evolution of radio AGN out to $z=1.3$. In Section 5, we analyze the properties of weak and powerful radio AGNs at low and intermediate redshifts; in Section 6 we compute the radio AGN mass function, and derive and compare the star formation quenching and radio AGN triggering rates. We discuss our results in the context of galaxy formation and evolution in Section 7, and summarize them in Section 8.

We report magnitudes in the $\mathrm{AB}$ system, adopt $H_{0}=$ $70, \Omega_{M}=0.3, \Omega_{\Lambda}=0.7$, and define the radio synchrotron spectrum as $F_{v} v^{-\alpha}$, assuming $\alpha=0.7$. 


\section{THE VLA-COSMOS RADIO AGN SAMPLE}

The sample of AGN galaxies used here is presented in Smolčić et al. (2008, S08 hereafter), and we briefly summarize it below.

Using radio and optical data for the COSMOS field, S08 have constructed a sample of 601 AGN galaxies with $z \leqslant 1.3$ from the entire VLA-COSMOS large project catalog (Schinnerer et al. 2007). The selection required optical counterparts with $i_{\mathrm{AB}} \leqslant 26$, accurate photometry, and an $\mathrm{S} / \mathrm{N} \geqslant 5$ (i.e., $\gtrsim 50 \mu \mathrm{Jy}$ ) detection at $20 \mathrm{~cm}$, and is based on a rest-frame optical color classification (see also Smolčić et al. 2006). The classification method was well calibrated using a large local sample of galaxies ( 7,000 Sloan Digital Sky Survey (SDSS) “main" spectroscopic galaxy sample, NRAO VLA Sky Survey (NVSS) and IRAS surveys) representative of the VLA-COSMOS population. It was shown that the method agrees well with other independent classification schemes based on mid-infrared colors (Lacy et al. 2004; Stern et al. 2005) and optical spectroscopy (Baldwin et al. 1981; Best et al. 2005). The selected sample of AGN is estimated to be $\sim 90 \%$ complete.

The rest-frame color classification procedure efficiently selects mostly type 2 AGNs (such as LINER, Seyferts), and absorption-line AGNs (with no emission lines in the optical spectrum), while type 1 AGNs (i.e., quasars, $\lesssim 20 \%$ of the total AGNs sample) are not included in the current sample (see S08 for detailed definitions of the samples).

Although based on a color identification (as opposed to an optical spectroscopic classification), the output of the selection of our intermediate redshift $(z \leqslant 1.3)$ AGNs sample is comparable to those of numerous local $(z \leqslant 0.3)$ radio AGN samples extensively studied in the literature (e.g., Sadler et al. 2002; Best et al. 2005; Mauch \& Sadler 2007, drawn from the SDSS, $2 \mathrm{dF}$, and $6 \mathrm{dF}$ optical surveys combined with the NVSS and FIRST $20 \mathrm{~cm}$ radio surveys). This is an important feature as it enables a straightforward and fair comparison of our results with those based on these local studies (e.g., the local AGNs radio-LF).

Out of the 601 selected AGN galaxies 262 have spectroscopic redshifts, while the remaining sources have very reliable photometric redshifts available $\left(\sigma\left(\frac{\Delta z}{1+z}\right)=0.027\right.$; see S08 and references therein). Based on Monte Carlo simulations, S08 have shown that the photometric errors in the rest-frame color introduce a small, $\sim 5 \%$, uncertainty in the number of the selected galaxies. Here we use the S08 sample of AGN galaxies, statistically corrected for this effect.

\section{THE 1.4 GHZ LUMINOSITY FUNCTION FOR RADIO AGN IN VLA-COSMOS}

\subsection{Derivation of the $L F$}

We derive the radio luminosity function $(\Phi)$ for our AGN galaxies in four redshift bins using the standard $1 / V_{\max }$ method (Schmidt 1968). We limit the accessible volumes (1) on the bright end by the minimum redshift cut-off of the redshift range in consideration or the minimum redshift out to which an object could be observed due to the optical saturation limit of $i^{*}=16$ (AB mag; see also Capak et al. 2007), and (2) on the faint end by the maximum redshift out to which a galaxy could be observed given the flux limits on both the radio and optical data. In practice, the latter is dominated by the radio detection limits, rather than the optical, as the major fraction of the sources used here has $i_{\mathrm{AB}}$ band magnitudes brighter than $\sim 24$ (see Figure 21 in S08).
We further take into account the nonuniform rms noise level in the VLA-COSMOS mosaic via the differential visibility area (i.e., areal coverage, $A_{k}$, versus rms; see Figure 13 in Schinnerer et al. 2007). Hence, for a source with a $1.4 \mathrm{GHz}$ luminosity $L_{j}$ its maximum volume is $V_{\max }\left(L_{j}\right)=\sum_{k=1}^{n} A_{k} \cdot V_{\max }\left(z_{\max }^{A_{k}}, L_{j}\right)$.

In order to robustly derive the LF we take several additional corrections into account: (1) the VLA-COSMOS detection completeness (Bondi et al. 2008), and (2) the AGN galaxy selection bias due to the rest-frame color uncertainties. We correct for these in the same way as described in Smolčić et al. (2009, S09 hereafter). The median of the first correction (as a function of flux density) is $\sim 10 \%$ (reaching a maximum value of $60 \%$ in only one of the lowest flux density bins; see Table 1 in Bondi et al. 2008). The second correction, based on Monte Carlo simulations, yields a reduction of $\sim 5 \%$ of the average number of radio AGN (see below; see also S09 for a more detailed description).

Hence, in the $i^{\text {th }}$ luminosity bin the comoving space density $\left(\Phi_{i}\right)$, and its corresponding error $\left(\sigma_{i}\right)$, are computed by weighting the contribution of each $\left(\mathrm{j}^{\text {th }}\right)$ galaxy by the completeness correction factor, $f_{\text {det }}$ (see Bondi et al. 2008):

$$
\Phi_{i}=\sum_{j=1}^{N} \frac{f_{\mathrm{det}}^{j}}{V_{\max }^{j}} ; \quad \sigma_{i}=\sqrt{\sum_{j=1}^{N}\left(\frac{f_{\mathrm{det}}^{j}}{V_{\max }^{j}}\right)^{2}} .
$$

The selection bias due to the rest-frame color uncertainties is accounted for via Monte Carlo simulations. As described in S08 and S09, in each iteration the rest-frame color error distribution is generated (see Figure 5 in S08) and added to the rest-frame color derived by spectral energy distribution (SED) fitting. AGN galaxies are then reselected, and the LF is derived as described above. In this way we obtain 100 realizations of $\left(\Phi_{i}, \sigma_{i}\right)$ for each luminosity bin, and we take the median values as representative.

\subsection{The Radio AGN Luminosity Function}

The $1.4 \mathrm{GHz}$ radio LFs for our AGN galaxies for the four chosen redshift bins are shown in Figure 1, and tabulated in Table 1. In each panel in Figure 1 we also show the local $20 \mathrm{~cm}$ LFs for AGNs derived by Condon et al. (2002), Sadler et al. (2002), Best et al. (2005), and Mauch \& Sadler (2007). Our derived LF in the local redshift bin (top left panel) agrees well with the local LFs. This is quite remarkable as (1) our identification method is based only on photometry contrary to the spectroscopic selection performed by the other studies (Sadler et al. 2002; Best et al. 2005; Mauch \& Sadler 2007), and (2) the $2 \square^{\circ}$ COSMOS field samples a significantly smaller comoving volume at these low redshifts compared to the almost all-sky surveys used by the other studies. This verifies that both our selection method, as well as the derivation of the LF are correct. Within the errors our lowest redshift LF seems to be best represented by the local LF derived by Sadler et al. (2002, Sad02 hereafter), and we adopt this local LF for further analysis of the evolution of the population of our AGNs.

In the top right panel, in Figure 1, we compare our derived AGN LF $(0.35<z \leqslant 0.6)$ with the volume densities computed by Sadler et al. (2007) for a redshift range $0.4<z<0.7$ based on 2SLAQ Luminous Red Galaxy Survey and NVSS, FIRST data. We convert their data to a base of $d \log L$, and scale them to match the mean redshift of our redshift bin $(z=0.475$ compared to their $z=0.55$ ) using pure luminosity evolution as obtained by Sadler et al. (2007, note that this only decreases their 


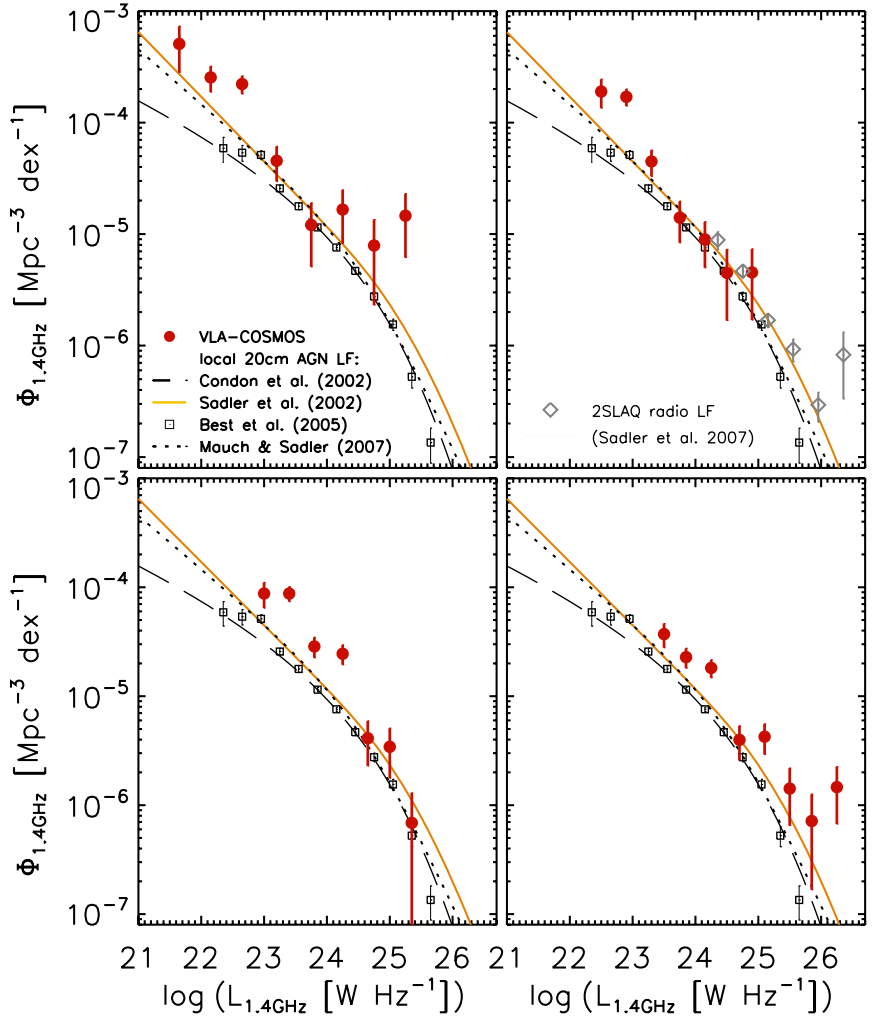

Figure 1. $1.4 \mathrm{GHz}$ LFs for AGN in the VLA-COSMOS survey (filled red circles), shown for four redshift ranges indicated in each panel. The local AGN volume densities derived by Condon et al. (2002, dashed black line), Sadler et al. (2002, solid orange line), Best et al. (2005, empty squares), and Mauch \& Sadler (2007, dotted line) are also plotted in each panel. The empty symbols in the top right panel are the radio AGN volume densities derived for the redshift range $0.4<z<0.7$ by Sadler et al. (2007) for the 2SLAQ Luminous Red Galaxy survey sample, and scaled here to match our mean redshift value of 0.475 (see the text for details).

(A color version of this figure is available in the online journal.)

radio luminosities by $10 \%$ ). The LFs are in excellent agreement. It is noteworthy that we well constrain the volume densities at the low-luminosity end $\left(\lesssim 10^{25} \mathrm{~W} \mathrm{~Hz}^{-1}\right)$, while the sample of Sadler et al. (2007) extends further out to $1.6 \times 10^{27} \mathrm{~W} \mathrm{~Hz}^{-1}$ (see also Figure 3, and Section 7).

The VLA-COSMOS AGN volume densities in the two highest redshift bins $(0.6<\mathrm{z} \leqslant 0.9$ and $0.9<z \leqslant 1.3)$ are shown in the bottom panels in Figure 1. The radio AGN LF at these redshifts has been studied in full detail only for higherpower AGN $\left(L_{1.4 \mathrm{GHz}}>10^{25} \mathrm{~W} \mathrm{~Hz}^{-1}\right.$; Dunlop \& Peacock 1990; Willot et al. 2001; Waddington et al. 2001), while constraining the low-luminosity end (sampled here) has been hampered by low number statistics due to small field sizes. Cowie et al. (2004) combined two deep $1.4 \mathrm{GHz}$ radio surveys of the Hubble Deep Field-North (40' in diameter; $5 \sigma \sim 40 \mu \mathrm{Jy}$ in the central region) and SSA13 (34' in diameter; $5 \sigma \sim 25 \mu \mathrm{Jy}$ in the center) fields, and derived the AGN radio LF in two separate redshift ranges similar to ours, and at comparable luminosities to our work. Our results are in qualitative agreement with those derived by Cowie et al. (2004, see their Figure 3). However, their sample is a factor of $\sim 7$ smaller than that used here.

\section{THE EVOLUTION OF RADIO AGN}

In this section, we constrain the evolution of our low-power radio AGNs using the VLA-COSMOS AGN data (Section 4.1). We further extend this to high-power radio AGNs using the
Table 1

Luminosity Functions for VLA-COSMOS AGN

\begin{tabular}{|c|c|c|}
\hline $\begin{array}{l}\text { Redshift } \\
\text { Range }\end{array}$ & $\begin{array}{c}\mathrm{L}_{1.4 \mathrm{GHz}} \\
\left(\mathrm{W} \mathrm{Hz}{ }^{-1}\right)\end{array}$ & $\begin{array}{c}\Phi \\
\left(\mathrm{Mpc}^{-3} \mathrm{dex}^{-1}\right)\end{array}$ \\
\hline \multirow{8}{*}{$0.1<z \leqslant 0.35$} & $4.47 \times 10^{21}$ & $5.08 \pm 2.25 \times 10^{-4}$ \\
\hline & $1.41 \times 10^{22}$ & $2.54 \pm 0.66 \times 10^{-4}$ \\
\hline & $4.47 \times 10^{22}$ & $2.22 \pm 0.40 \times 10^{-4}$ \\
\hline & $1.58 \times 10^{23}$ & $4.54 \pm 1.57 \times 10^{-5}$ \\
\hline & $5.62 \times 10^{23}$ & $1.21 \pm 0.70 \times 10^{-5}$ \\
\hline & $1.78 \times 10^{24}$ & $1.66 \pm 0.88 \times 10^{-5}$ \\
\hline & $5.62 \times 10^{24}$ & $7.90 \pm 5.59 \times 10^{-6}$ \\
\hline & $1.78 \times 10^{25}$ & $1.46 \pm 0.84 \times 10^{-5}$ \\
\hline \multirow{7}{*}{$0.35<z \leqslant 0.6$} & $3.16 \times 10^{22}$ & $1.90 \pm 0.55 \times 10^{-4}$ \\
\hline & $7.94 \times 10^{22}$ & $1.70 \pm 0.29 \times 10^{-4}$ \\
\hline & $2.00 \times 10^{23}$ & $4.48 \pm 1.18 \times 10^{-5}$ \\
\hline & $5.62 \times 10^{23}$ & $1.40 \pm 0.57 \times 10^{-5}$ \\
\hline & $1.41 \times 10^{24}$ & $8.96 \pm 3.96 \times 10^{-6}$ \\
\hline & $3.16 \times 10^{24}$ & $4.49 \pm 2.81 \times 10^{-6}$ \\
\hline & $7.94 \times 10^{24}$ & $4.53 \pm 2.84 \times 10^{-6}$ \\
\hline \multirow{7}{*}{$0.6<z \leqslant 0.9$} & $1.00 \times 10^{23}$ & $8.76 \pm 2.32 \times 10^{-5}$ \\
\hline & $2.51 \times 10^{23}$ & $8.75 \pm 1.36 \times 10^{-5}$ \\
\hline & $6.31 \times 10^{23}$ & $2.86 \pm 0.61 \times 10^{-5}$ \\
\hline & $1.78 \times 10^{24}$ & $2.45 \pm 0.50 \times 10^{-5}$ \\
\hline & $4.47 \times 10^{24}$ & $4.11 \pm 1.82 \times 10^{-6}$ \\
\hline & $1.00 \times 10^{25}$ & $3.43 \pm 1.66 \times 10^{-6}$ \\
\hline & $2.24 \times 10^{25}$ & $6.88 \pm 7.45 \times 10^{-7}$ \\
\hline \multirow{8}{*}{$0.9<z \leqslant 1.3$} & $3.16 \times 10^{23}$ & $3.72 \pm 0.93 \times 10^{-5}$ \\
\hline & $7.08 \times 10^{23}$ & $2.28 \pm 0.47 \times 10^{-5}$ \\
\hline & $1.78 \times 10^{24}$ & $1.82 \pm 0.34 \times 10^{-5}$ \\
\hline & $5.01 \times 10^{24}$ & $3.97 \pm 1.38 \times 10^{-6}$ \\
\hline & $1.26 \times 10^{25}$ & $4.25 \pm 1.33 \times 10^{-6}$ \\
\hline & $3.16 \times 10^{25}$ & $1.42 \pm 0.77 \times 10^{-6}$ \\
\hline & $7.08 \times 10^{25}$ & $7.16 \pm 5.48 \times 10^{-7}$ \\
\hline & $1.78 \times 10^{26}$ & $1.46 \pm 0.79 \times 10^{-6}$ \\
\hline
\end{tabular}

Note. $\mathrm{H}_{0}=70, \Omega_{M}=0.3, \Omega_{\Lambda}=0.7$.

model obtained by Willott et al. (2001) based on high-power AGN samples drawn from the 3CRR, 6CE, and 7CRS surveys (Section 4.2).

\subsection{The Evolution of Low-Power Radio AGN Galaxies in the COSMOS Field}

The evolution of an astrophysical population is usually parameterized by monotonic density and luminosity evolution of its local LF:

$$
\Phi_{z}(L)=(1+z)^{\alpha_{D}} \times \Phi_{z=0}\left[\frac{L}{(1+z)^{\alpha_{L}}}\right],
$$

where $\alpha_{D}$ and $\alpha_{L}$ are the characteristic density and luminosity evolution parameters, respectively, $L$ is the luminosity, $\Phi_{z}(L)$ is the LF at redshift $z$, and $\Phi_{z=0}$ is the local LF.

It is well known that strong degeneracy between luminosity and density evolution exists, even if the observational data sample the turnover ("knee") of the LF at different cosmological times (see, e.g., Le Floc'h et al. 2005). The VLA-COSMOS AGN sample in particular lacks high-luminosity objects that could constrain the turnover of the LF in all our redshift bins (see Figure 1). Hence, we do not attempt to try to break the luminosity-density evolution degeneracy, but we separately constrain both pure density (PDE; $\alpha_{L}=0$ ) and pure luminosity (PLE; $\alpha_{D}=0$ ) evolution based on our data. We adopt the Sad02 local AGN LF $\left(\Phi_{z=0}\right)$ as the representative LF in the 


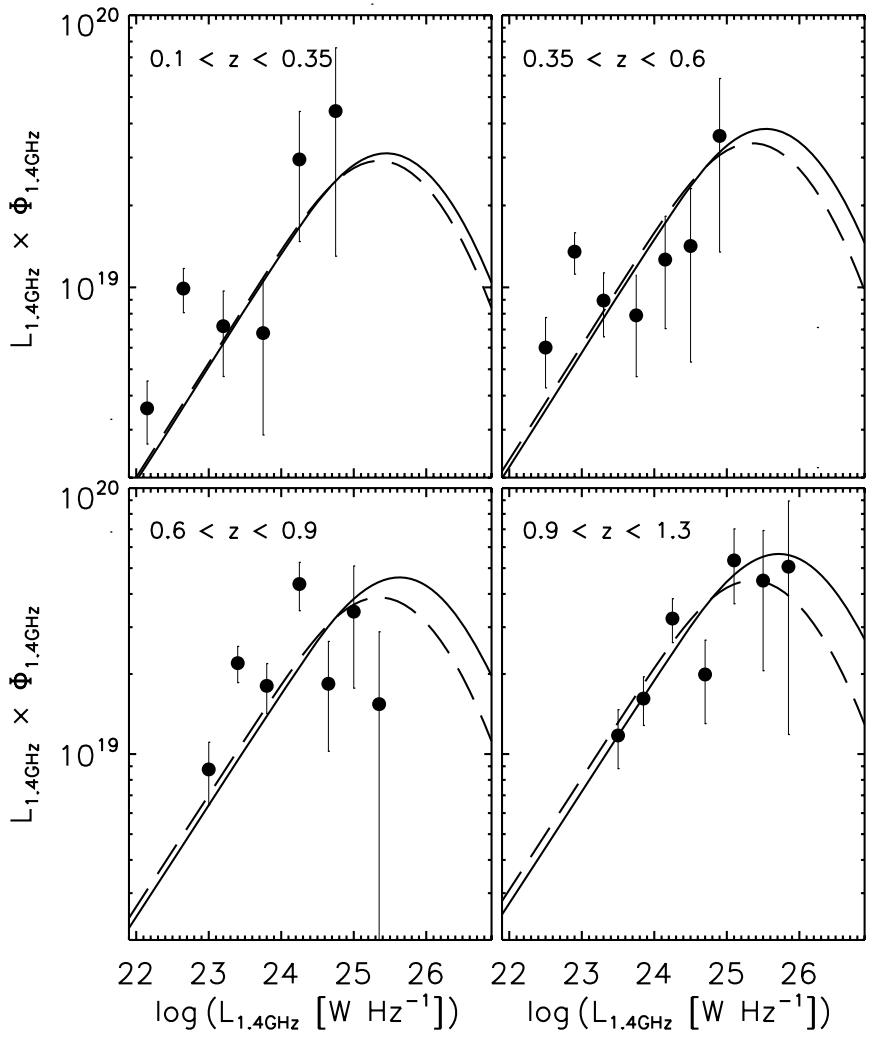

Figure 2. Luminosity density for the VLA-COSMOS AGN in four redshift ranges (filled circles). The lines represent the best fit pure density (dashed line) and pure luminosity (solid line) evolution of the local $20 \mathrm{~cm}$ AGN LF (see the text for details).

local universe, which is given by the following analytical form:

$$
\Phi(L)=\Phi^{*}\left[\frac{L}{L_{*}}\right]^{1-\alpha} \exp \left\{-\frac{1}{2 \sigma^{2}}\left[\log \left(1+\frac{L}{L_{*}}\right)\right]^{2}\right\},
$$

where $\alpha=1.58, \sigma=1.0, \Phi^{*}=7.6 \times 10^{-6} \mathrm{Mpc}^{-3}$, and $L^{*}=2.1 \times 10^{24} \mathrm{~W} \mathrm{~Hz}^{-1}$ for their AGN population (scaled to the cosmology used here, and to the base of $d \log L$ ).

For both PDE and PLE we derive the evolution by summing the $\chi^{2}$ distributions obtained for a large range of evolution parameters $\alpha_{L}$ and $\alpha_{D}\left(\left(\alpha_{L}, 0\right)\right.$ for PLE; $\left(0, \alpha_{D}\right)$ for PDE) in each particular redshift bin (excluding our first local redshift bin). The uncertainty in $\alpha$ is then taken to be the $1 \sigma$ error obtained from the $\chi^{2}$ statistics. Our results yield a pure density evolution with $\alpha_{D}=1.1 \pm 0.1$, or alternatively a pure luminosity evolution with $\alpha_{L}=0.8 \pm 0.1$.

In Figure 2 we show the luminosity density for our AGN in the four redshift ranges defined in Figure 1. We also plot the luminosity density given the derived PD and PL evolutions (lines). From the figure it becomes obvious that we cannot distinguish between these two types of evolution given our data, as our sample, tracing only the lower-luminosity end of the LF, appears to be described equally well by both. Thus, in the further analysis we will take the range of the two best-fit evolution models (taking also their errors into account) as representative of the range of uncertainties.

In summary, our results imply either a PLE with $L_{*} \propto$ $(1+z)^{0.8 \pm 0.1}$ or a PDE where $\Phi_{*} \propto(1+z)^{1.1 \pm 0.1}$. Regardless of which model is physically more appropriate to describe the real cosmic evolution of the VLA-COSMOS AGN population, both imply a modest evolution of radio-luminous AGN in the luminosity range of $\sim 10^{22}-10^{25} \mathrm{~W} \mathrm{~Hz}^{-1}$.

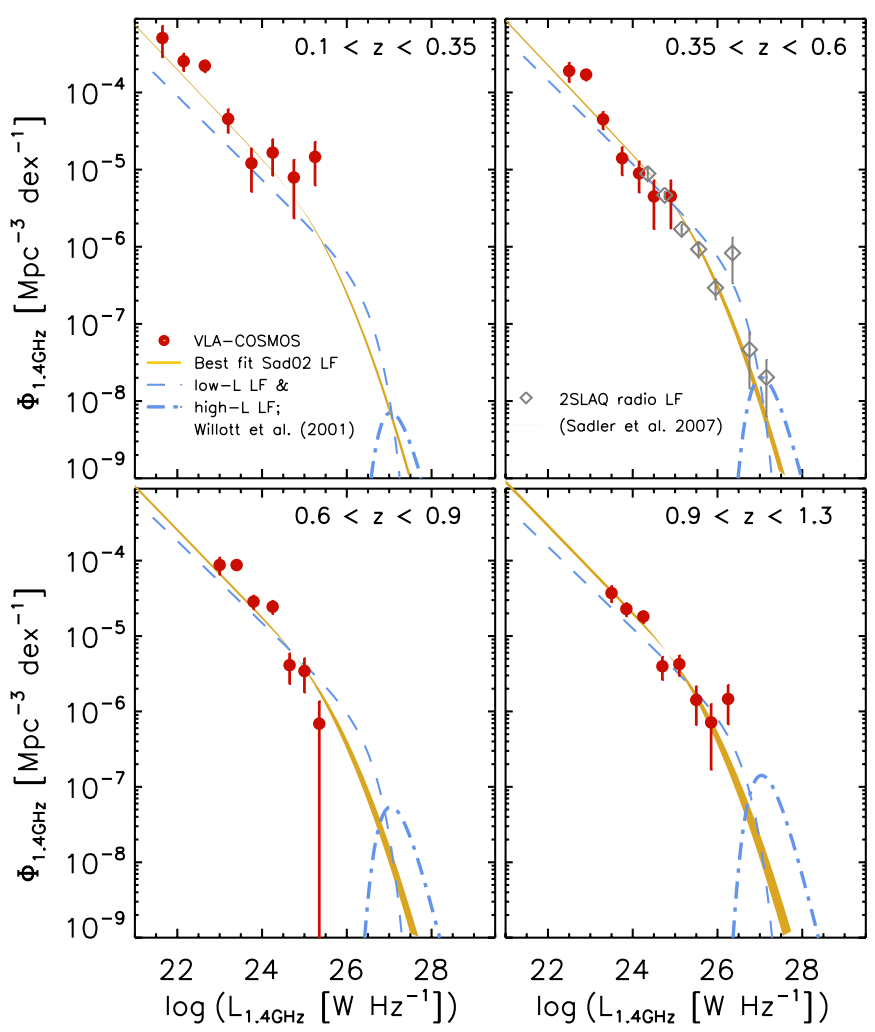

Figure 3. VLA-COSMOS AGN volume densities at $20 \mathrm{~cm}$ in four redshift ranges (red filled circles; analogous to Figure 1). Also shown in each panel is our best-fit evolution corresponding to the range given by pure density and PLE of the Sad02 local LF and their corresponding errors (orange shaded curve), as well as the radio AGN LF model given by Willott et al. (2001, model 'C') for their less luminous population (dashed blue curves), and high-luminosity population (dashed-dotted blue curves; scaled to current cosmology and $1.4 \mathrm{GHz}$ radio frequency; see the text for details). In the top right panel the volume densities derived by Sadler et al. (2007, scaled to match our mean redshift value of 0.475; see also Figure 1) are shown.

(A color version of this figure is available in the online journal.)

Our results are consistent with previous findings. Based on a $V / V_{\max }$ analysis out to a redshift of $\sim 1$, Clewley \& Jarvis (2004) have found no strong evolution of low-radio-luminosity sources $\left(L_{325 \mathrm{MHz}} \lesssim 10^{25} \mathrm{~W} \mathrm{~Hz} \mathrm{sr}^{-1}\right.$, corresponding to $L_{1.4 \mathrm{GHz}} \lesssim 4.5 \times 10^{\widetilde{25}} \mathrm{~W} \mathrm{~Hz}^{-1}$ ) at least up to $z \sim 0.6$. Further, Sadler et al. (2007) have constrained the evolution of the LF for radio-luminous $\mathrm{AGN}\left(L_{1.4 \mathrm{GHz}} \sim 10^{24}-10^{27} \mathrm{~W} \mathrm{~Hz}^{-1}\right)$ out to $z \sim 0.7$. Using the local AGN LF given by Mauch \& Sadler (2007), they have found a PLE with $L_{*} \propto(1+z)^{2.0 \pm 0.3}$. The VLA-COSMOS AGN LF in the redshift range $0.35<z \leqslant 0.6$, and the Sadler et al. (2007) AGN LF $(0.4<z<0.7)$ agree remarkably well (see top right panel in Figures 1 and 3). If we parameterize the evolution of the VLA-COSMOS AGN in the same way as described in Sadler et al. (2007, using the same local LF), we derive a PLE of $1.2 \pm 0.4$ for the $0.35<z \leqslant 0.6$ redshift bin. It has to be noted, however, that the VLA-COSMOS and the 2SLAQ samples constrain different radio luminosity ranges (see Figure 3). Our results agree within the uncertainties with those of Mauch \& Sadler (2007), however they on average imply a slower evolution. This is consistent with a more modest evolution of low radio power, compared to high radio power, radio AGN (Willott et al. 2001; Waddington et al. 2001; Clewley \& Jarvis 2004). Our findings are also in very good agreement with the recent results based on NVSS/FIRST and the MegaZLuminous Red Galaxy catalog drawn from the SDSS (Donoso et al. 2008). 


\subsection{The Evolution of High-Power Radio AGN}

In order to quantify the contribution of different populations to the comoving radio energy density at different cosmic times, all radio AGN populations need to be considered. As low- and high- power radio AGN seem to evolve in a different manner (see Section 1) and our VLA-COSMOS AGN sample only the lowpower radio AGN, we need to make the following assumptions about the evolution of the high-power radio sources.

Based on the 3CRR, 6CE, and 7CRS radio surveys combined with complete optical spectroscopy, Willott et al. (2001) have successfully modeled the radio AGN LF using two radio populations-a less powerful $\left(L_{1.4 \mathrm{GHz}} \sim 2.5 \times 10^{25-27} \mathrm{~W}\right.$ $\mathrm{Hz}^{-1}$ ) population comprising both FR I and FR II sources, and a powerful population $\left(L_{1.4 \mathrm{GHz}} \gtrsim 2.5 \times 10^{26} \mathrm{~W} \mathrm{~Hz}^{-1}\right)$ comprising mostly FR II sources. They have modeled the evolution of the first population as a pure density evolution up to a maximum redshift $(\sim 0.7)$ beyond which any evolution ceases. The evolution of the powerful population has been assumed to change in density following a Gaussian distribution in redshift, which was allowed to have a different shape beyond its redshift peak at $z \sim 2$ (see Table 1 in Willott et al. 2001). It is worth noting that the Willott et al. (2001) model agrees well with the Dunlop \& Peacock (1990) steep-spectrum model.

The VLA-COSMOS AGN sample constrains the faint end of the radio AGN LF, and here we use the Willott et al. (2001) model to extend our radio LF to high powers. In Figure 3 we compare our LFs with the Willott et al. (2001) model, after the latter has been converted to the current cosmology and the $151 \mathrm{MHz}$ radio luminosities scaled to $1.4 \mathrm{GHz}$. The VLACOSMOS AGN data and the Sad02 LF constrain the radio LF more robustly at the faint end compared to the Willott et al. (2001) model for their less-powerful radio AGN. Thus, in the further analysis we will constrain the low-power radio AGN LF and its evolution using the VLA-COSMOS sample as described in the previous section, and we will use only the powerful radio AGN model by Willott et al. (2001) to describe the evolution of high-radio-power AGN (see dashed-dotted curves in Figure 3).

\subsection{The Evolution of the Comoving Radio-Luminosity Density for AGN Galaxies}

At a specific cosmic time the integrated comoving luminosity density represents the total power per unit comoving volume of a given astronomical population. Thus, if divided into distinct populations of objects it traces their relative contribution to the overall power output at a given redshift. To estimate the contribution of low and high-radio-power AGN to the AGN radio luminosity output at different cosmic times, we investigate in the following the evolution of the comoving $1.4 \mathrm{GHz}$ luminosity density, $\Omega_{1.4 \mathrm{GHz}}$, for our VLA-COSMOS AGN $\left(L_{1.4 \mathrm{GHz}} \lesssim 10^{25} \mathrm{~W} \mathrm{~Hz}{ }^{-1}\right)$ and the high-power AGN $\left(L_{1.4 \mathrm{GHz}} \gtrsim 10^{26} \mathrm{~W} \mathrm{~Hz}{ }^{-1}\right)$, adopting the Willott et al. (2001) model.

For a given redshift, $\Omega_{1.4 \mathrm{GHz}}$ has been computed by integrating the luminosity density over the entire range of radio luminosities. For the VLA-COSMOS AGN the luminosity density has been constrained using our best-fit PLE and PDE models (see curves in Figure 2 and Section 4.1), and for the high-power population using the Willott et al. (2001) model "C", with the corresponding errors (see Table 1 in Willott et al. 2001 and Section 4.2).

In Figure 4, we show the evolution of the comoving $20 \mathrm{~cm}$ integrated luminosity density for all radio AGN as well as the

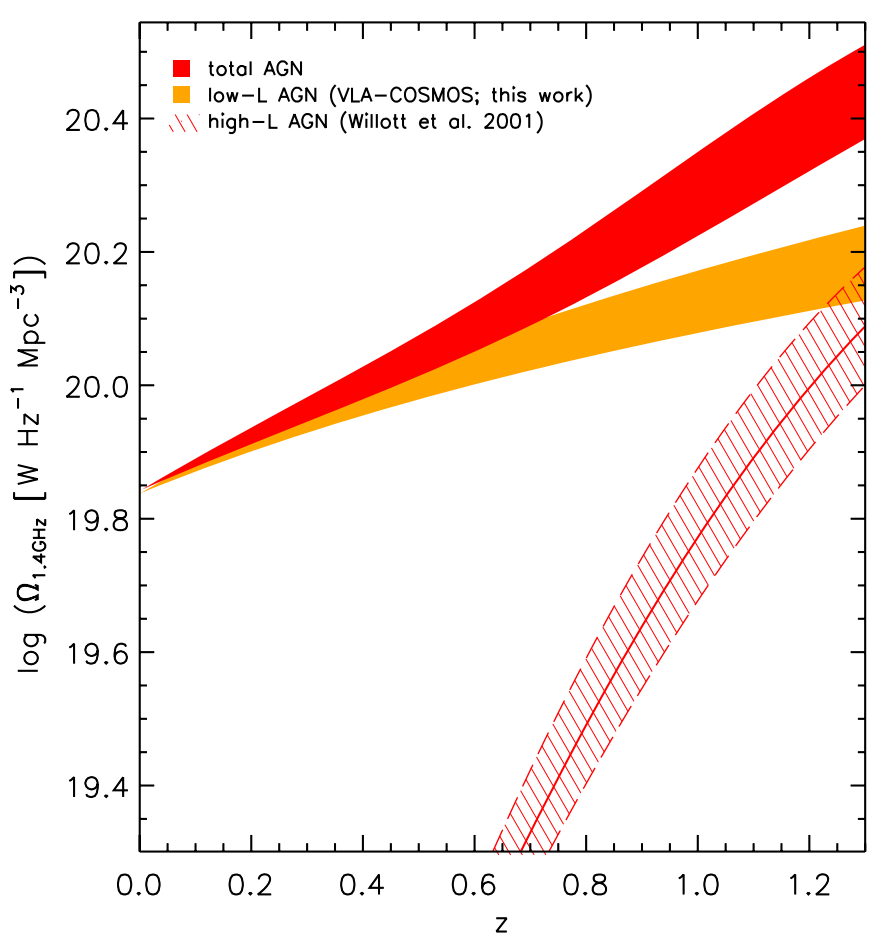

Figure 4. Evolution of the comoving $20 \mathrm{~cm}$ integrated luminosity density for VLA-COSMOS AGN (orange curve) galaxies since $z=1.3$. Shown is also the evolution of the high-luminosity radio AGN, adopted from Willott et al. (2001, hatched region; the thick and dashed lines correspond to the mean, maximum and minimum results, respectively). The evolution for the total AGN population, obtained by co-adding the VLA-COSMOS and high-luminosity AGN energy densities, is shown as the red-shaded curve (see the text for details).

(A color version of this figure is available in the online journal.)

low and high-radio-power AGN separately. The evolution of these two populations is very different; high-power AGN evolve significantly stronger than low-power AGN. The total $\Omega_{1.4 \mathrm{GHz}}$ for radio AGN is dominated by low-power AGN at low redshifts $(z \lesssim 0.7)$ where the contribution of high-power radio sources is negligible. However, at $z \gtrsim 0.7$ high-power sources begin to contribute significantly to the overall integrated luminosity density, and at a redshift of $\sim 1.3$ their contribution to the total AGN $\Omega_{1.4 \mathrm{GHz}}$ is comparable to the one of low-power AGN. The implications of these populations for galaxy formation and evolution, also out to higher redshifts $(z=2.5)$, are discussed in Section 7.

\section{PROPERTIES OF RADIO AGN}

In this section we outline and compare the properties of low and high-radio-power AGN in the local (Section 5.1) and intermediate-redshift (Section 5.2) universe. We find that already by $z \sim 1$ the host galaxies of low-power, VLA-COSMOS AGN have built-up stellar and $\mathrm{BH}$ masses comparable to the highest mass galaxies observed locally. As their BH masses are already significant at these intermediate redshifts, this implies that they will no longer be able to have a phase of high accretion (i.e., vigorous $\mathrm{BH}$ growth), consistent with numerous previous studies of similar samples (e.g., Allen et al. 2006; Evans et al. 2006; Hardcastle et al. 2006, 2007). For the high-radio-power AGN on the other hand, extensive evidence exists in the literature that they accrete at high rates-representing a mode of substantial BH growth. 
Table 2

Properties of LERAGN and HERAGN in the Local Universe

\begin{tabular}{|c|c|c|c|c|}
\hline Property & LERAGN & Ref. & HERAGN & Ref. \\
\hline Object class & mainly FR I & $(1),(2)$ & mainly FR II & $(1),(2)$ \\
\hline Radio luminosity & $L_{1.4 \mathrm{GHz}} \lesssim 10^{25} \mathrm{~W} \mathrm{~Hz}^{-1}$ & (3) & $L_{1.4 \mathrm{GHz}} \gtrsim 10^{25} \mathrm{~W} \mathrm{~Hz}^{-1}$ & (3) \\
\hline Density environment & moderate-to-high & $(4),(5)$ & moderate-to-low & (4) \\
\hline Optical morphology & regular & $(8),(9)$ & often distorted & (8), (9), (10) \\
\hline Optical color & red & (9), (11) & bluer (compared to LERAGN) & $(10),(9)$ \\
\hline Stellar mass & highest $\left(\gtrsim 5 \times 10^{10} M_{\odot}\right)$ & $(3),(11)$ & lower (compared to LERAGN) & $(3),(12)$ \\
\hline BH mass & highest $\left(\sim 10^{9} M_{\odot}\right)$ & (9), (13) & lower (compared to LERAGN) & (3), (14) \\
\hline ISM content & low & (15), (16), (17) & higher (compared to LERAGN) & $(15),(16),(17),(18),(19)$ \\
\hline Accretion mode & radiatively inefficient & $(1),(2),(14),(20)$ & radiatively efficient & $(1),(2),(20),(21),(22)$ \\
\hline
\end{tabular}

Notes. References: (1) Evans et al. 2006; (2) Hardcastle et al. 2006; (3) Kauffmann et al. 2008; (4) Baum et al. 1992; (5) Best et al. 2005; (8) Heckman et al. 1986; (9) Baum et al. 1992; (10) Baldi \& Capetti 2008; (11) Smolčić et al. 2008; (12) Tasse et al. 2008; (13) this work; (14) Chiaberge et al. 2005; (15) Leon et al. 2001; (16) de Koff et al. 1996; (17) Müller et al. 2004; (18) Evans et al. 2006; (19) Emonts et al. 2007; (20) Hardcastle et al. 2007; (21) Barthel 1989; (22) Haas 2004.

\subsection{Local Universe}

Various correlations are found in the literature between the presence of emission lines in AGN and their e.g., radio power, BH and stellar mass, as well as environment and the galaxies' gas content. We outline these below.

First, almost all FR I low-power radio galaxies are LERAGN, while optical hosts of FR IIs, which are typically more powerful than FR Is (Fanaroff \& Riley 1974; Owen 1993; Ledlow \& Owen 1996), usually have strong emission lines. ${ }^{19}$ Recently, based on a large statistically significant sample of local radio-optical sources (SDSS-NVSS-FIRST) Kauffmann et al. (2008) have found that the fraction of radio AGN whose optical hosts have emission lines in their spectra (predominantly HERAGN) is a strong function of radio luminosity. This emission-line galaxy fraction is roughly constant ( $\sim 40 \%)$ up to $L_{1.4 \mathrm{GHz}} \sim 10^{25} \mathrm{~W}$ $\mathrm{Hz}^{-1}$, beyond which it steeply rises approaching $\sim 80 \%$ at $\sim 4 \times 10^{25} \mathrm{~W} \mathrm{~Hz}^{-1}$ (see Figure 3 in Kauffmann et al. 2008). This "critical" luminosity, observed by Kauffmann et al., is remarkably close to the FR I-II break luminosity, as well as to the power which roughly separates the radio sources which show strong cosmological evolution from those which do not (see, e.g., Figure 4 and Section 4). Furthermore, based on the results of Kauffmann et al., the luminosity of $L_{1.4 \mathrm{GHz}} \sim 10^{25} \mathrm{~W} \mathrm{~Hz}^{-1}$ can be thought of as a rough threshold between HERAGN and LERAGN. Thus, most of the low-radio-power $\operatorname{AGN}\left(L_{1.4 \mathrm{GHz}} \lesssim\right.$ $10^{25} \mathrm{~W} \mathrm{~Hz}^{-1}$ ) are LERAGN, while the majority of powerful radio AGNs $\left(L_{1.4 \mathrm{GHz}} \gtrsim 10^{25} \mathrm{~W} \mathrm{~Hz}^{-1}\right)$ are HERAGN.

On the other hand, the fraction of emission line (i.e., HE) radio AGN in the local universe strongly decreases as a function of both stellar mass and velocity dispersion (see Figure 3 in Kauffmann et al. 2008). This implies that, at least locally, HE (or alternatively high-radio-power) AGN tend to have lower stellar masses, as well as lower BH masses compared to LERAGN. Even further, the latter constitute the most massive galaxies in the universe $\left(M_{\odot}>10^{11} M_{\odot}\right)$ that preferentially occupy the centers of high-galaxy-density regions (Baum et al. 1992; Best et al. 2005).

Furthermore, various studies in the literature have shown that HERAGN, contrary to LERAGN, tend to show unusually blue off-nuclear continuum colors (Baum et al. 1992), evidence of recent star formation (Baldi \& Capetti 2008), and often have distorted optical morphologies suggesting that they have

\footnotetext{
19 Note, however, that the correspondence between the FR class and the presence of emission lines is not exactly one-to-one.
}

undergone a recent major merger (Heckman et al. 1986; Baum et al. 1992; Baldi \& Capetti 2008). CO and H I observations of radio galaxies suggest larger amounts of cold gas in powerful, compared to low-power, radio sources (see, e.g., Figure 8 in Evans et al. 2005; see also Emonts et al. 2007), and there is evidence supporting quantitatively larger amounts of dust, and therefore gas (Leon et al. 2001; Solomon \& Vanden Bout 2005) in HERAGN compared to LERAGN (de Koff et al. 2000; Müller et al. 2004). A summary of the properties of radio AGN in the nearby universe is given in Table 2 .

\subsection{Intermediate Redshift}

In this section, we investigate the properties of low- and high-radio-power AGN at intermediate redshifts, and compare these to the properties of their local counterparts. In Section 3 and Section 4 we have derived the $1.4 \mathrm{GHz}$ radio $\mathrm{LF}$, and its evolution, for a sample of $\sim 600$ radio-luminous AGN $(0.1<z<1.3)$ drawn from the VLA-COSMOS survey. One of the main characteristics of this radio AGN sample is that it consists of predominantly (96\%) low-power AGN with $L_{1.4 \mathrm{GHz}} \lesssim 10^{25} \mathrm{~W} \mathrm{~Hz}^{-1}$. Ledlow \& Owen (1996) have shown that the threshold between weak FR I types of galaxies and powerful FR IIs is a function of the host galaxy's optical $(R$ band) magnitude, $M_{R}$. Hence, to better constrain the properties of the VLA-COSMOS AGN in Figure 5 we plot them in the $L_{1.4 \mathrm{GHz}}$ versus $\mathrm{M}_{\mathrm{R}}$ plane. Almost all (98\%) of our AGNs occupy the weak radio AGN, FR I region in this plane.

In order to get a further insight into the composition of the VLA-COSMOS AGN, we make use of the sources with available optical spectroscopy. Trump et al. (2007) have carried out a spectroscopic survey of X-ray and radio selected AGN candidates in the COSMOS field (using the Magellan/IMACS instrument). They have classified their sources into (1) broad emission line AGNs ("bl"), (2) narrow emission line AGNs ("nl"), (3) red galaxies with only absorption features in their spectrum ("e") and (4) hybrid objects showing a mix of narrow emission lines and a red galaxy continuum shape ("nle"). Combining a radio AGN detection with this classification scheme, we can very roughly consider the red and hybrid galaxy spectroscopic classification ("e+nle") as a proxy for LERAGN, and the narrow emission line AGN classification ("nl") as a proxy for HERAGN. About 35\% of our VLA-COSMOS AGNs have an available classification based on IMACS spectroscopy, and in Figure 6 the fractions of "e+nle" and "nl" galaxies are shown as a function of both redshift (left panel) and radio 


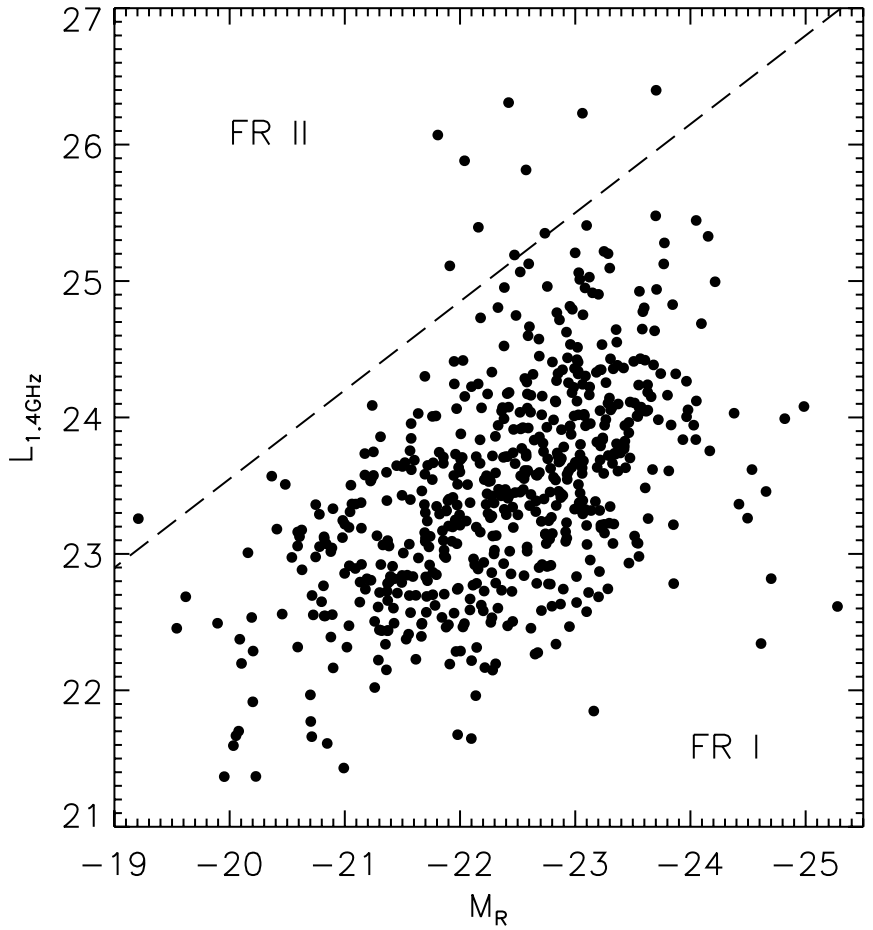

Figure 5. Monochromatic $1.4 \mathrm{GHz}$ radio power for VLA-COSMOS AGN as a function of their host galaxy absolute $R$-band magnitude. The dashed line corresponds to the separation between FR I and FR II types of galaxies given by Ledlow \& Owen (1996). Note that almost all of the VLA-COSMOS AGN occupy the low-power FR I region of this plane.

luminosity (right panel). The absorption line and hybrid galaxies dominate the VLA-COSMOS sample at a constant $\sim 80 \%$ level at all redshifts $(z \leqslant 1.3)$, while the narrow emission line AGNs contribute about $\sim 20 \%$ at all redshifts. There is an indication that in the highest redshift bin $(1<z<1.3)$, where our most luminous $\left(L_{1.4 \mathrm{GHz}}>10^{25} \mathrm{~W} \mathrm{~Hz}^{-1}\right)$ radio AGN are observed (see Figure 1), the fraction of narrow emission line AGNs rises. However, given the large error-bars in this redshift range (due to the low number of spectroscopically observed radio AGN) we cannot draw any robust conclusions regarding this. In the right panel of Figure 6 we show the fraction of 'e+nle' and 'nl' galaxies as a function of $1.4 \mathrm{GHz}$ power. Although the number of sources with $L_{1.4 \mathrm{GHz}} \gtrsim 10^{25} \mathrm{~W} \mathrm{~Hz}^{-1}$ is low in the VLACOSMOS AGN sample, there is an indication that the fraction of narrow-line objects (thus roughly HERAGN) increases beyond $\sim 10^{25} \mathrm{~W} \mathrm{~Hz}^{-1}$, consistent with the findings in the local universe (Kauffmann et al. 2008).

Assuming that the spectroscopic subsample represents well the full sample (note that this is a rather robust assumption as shown in S08; see their Figures 3 and 21), we conclude that LERAGN dominate the VLA-COSMOS radio AGN sample. Thus, similar to the findings in the local universe, our lowradio-power AGN at intermediate redshift are preferentially LERAGN.

Stellar masses, using a Chabrier (2003) initial mass function (IMF), have been computed for the entire VLA-COSMOS galaxy sample $(z \leqslant 1.3)$ by S08. In Figure 7 (top panels) we show the stellar masses for our VLA-COSMOS AGN as a function of redshift and $1.4 \mathrm{GHz}$ luminosity. The median stellar mass of our radio AGN is $1.6 \times 10^{11} M_{\odot}$, i.e., $\left\langle\log M_{*}\right\rangle=11.2$ (in any given redshift range) with a $1 \sigma$ scatter of $0.4 \mathrm{dex}$. This is consistent with the stellar masses of the most massive, local galaxies (e.g., Baldry et al. 2004; Best et al. 2005). In addition, it is worth noting that their average rest-frame colors are consistent with red galaxy colors (see, e.g., Figure 9 in S08).

We further compute the BH masses for the full VLACOSMOS AGN sample using the local correlation given by Marconi \& Hunt (2003; see also Graham 2007) which relates the $K$-band luminosity to the $\mathrm{BH}$ mass:

$$
\log _{10} M_{\mathrm{BH}}=8.21+1.13 \times\left(\log _{10} L_{K}-10.9\right),
$$

where $L_{K}$ is the rest-frame $K$-band luminosity (also in solar units). The scatter in the relation is 0.5 dex (Marconi \& Hunt 2003). The K-band rest-frame luminosity for our VLACOSMOS AGN was computed via SED fitting as described in detail in S08. In order to take into account passive luminosity
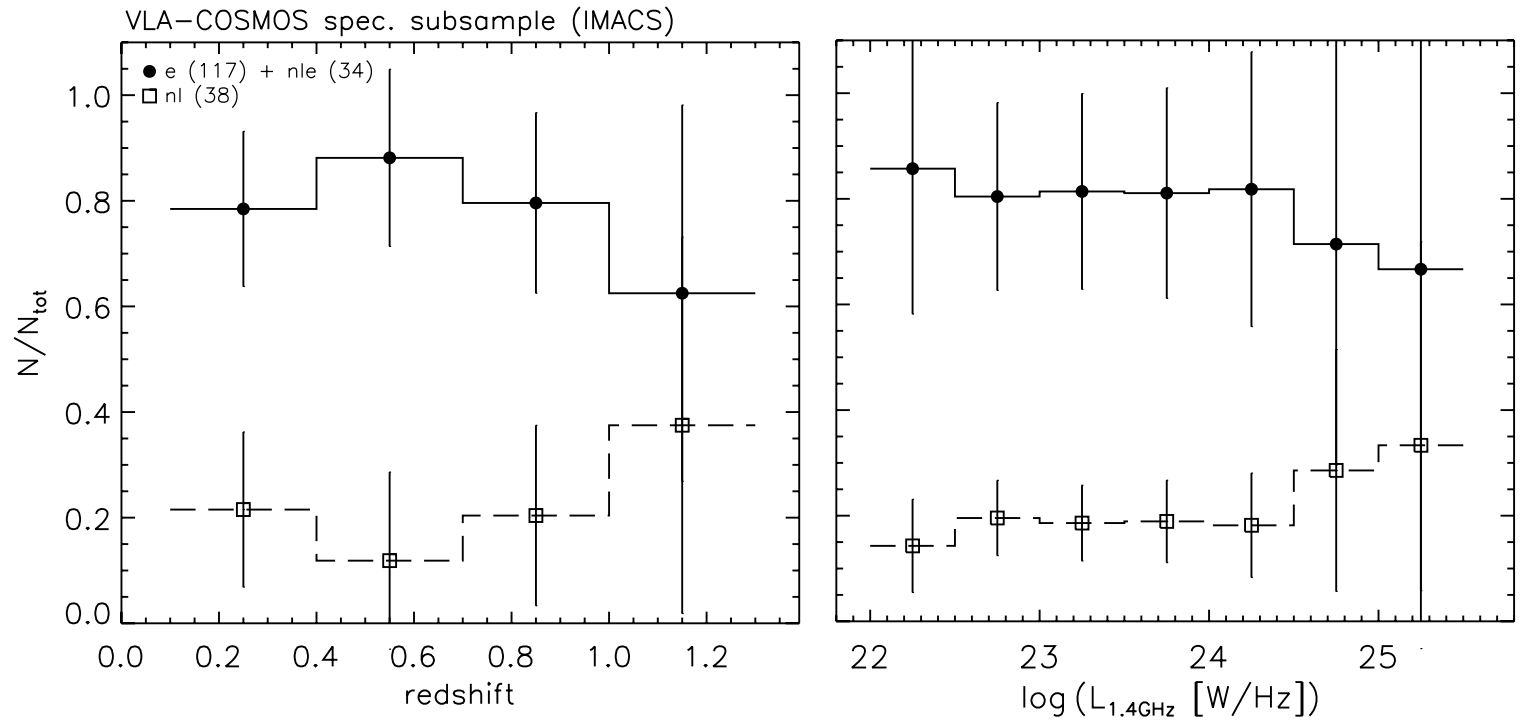

Figure 6. Fraction of VLA-COSMOS AGN with available Magellan/IMACS spectroscopy (Trump et al. 2007) as a function of redshift (left panel) and 1.4 GHz radio luminosity (right panel). The galaxies have been divided into two subsamples: (1) narrow emission line (nl) galaxies, and (2) elliptical galaxies (e) plus composite objects showing a red galaxy continuum and narrow emission lines (nle; see Trump et al. 2007 for details). These two subsamples roughly correspond to HE and LE AGN, respectively (see the text for details). The quantity and symbols for objects of each type is indicated in the left panel. Note that there is a slight (although noisy) trend showing that the fraction of narrow emission line objects (i.e., roughly HE AGN) increases as a function of both redshift and radio luminosity. 


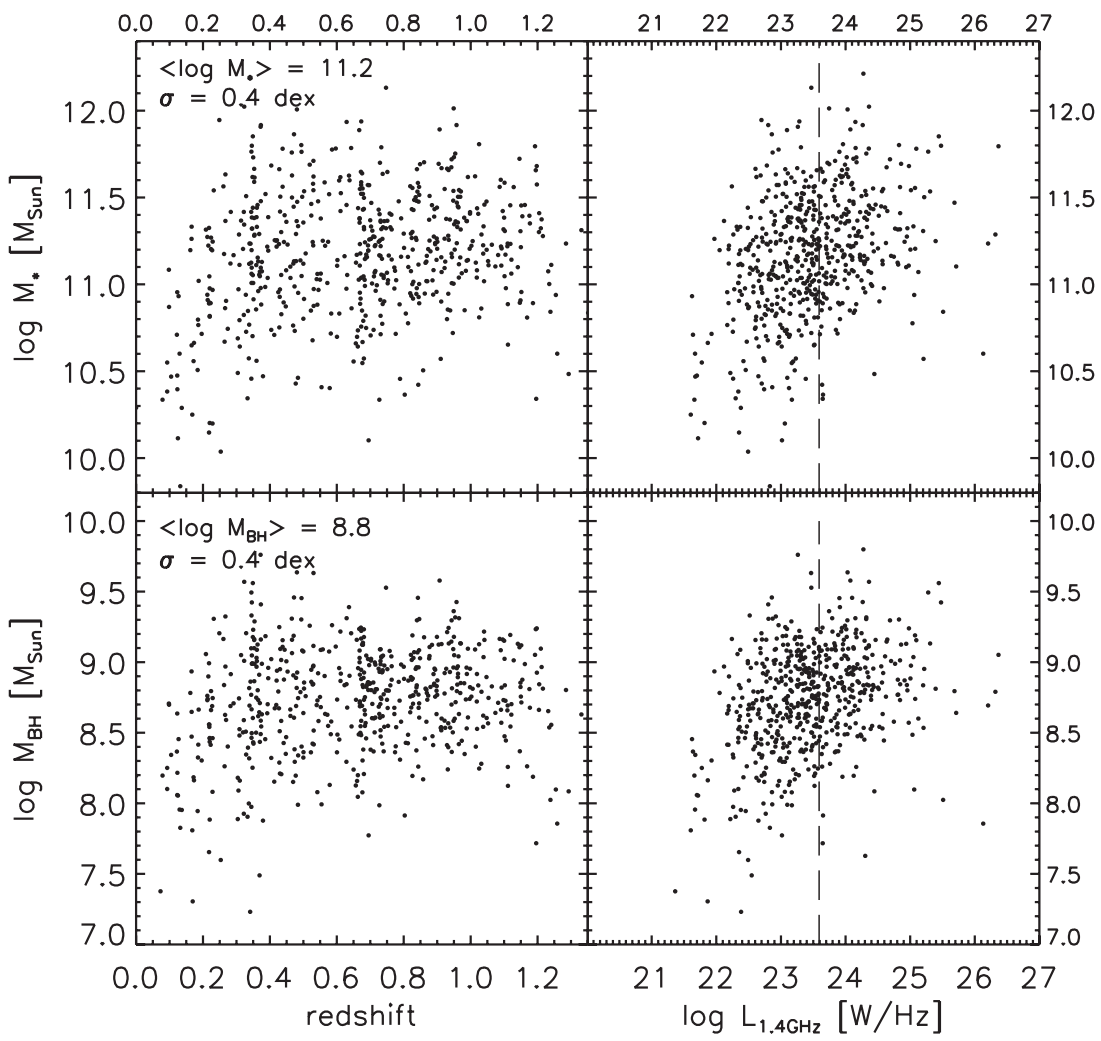

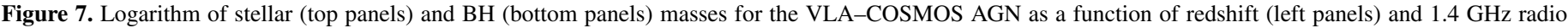

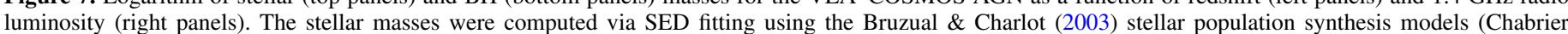

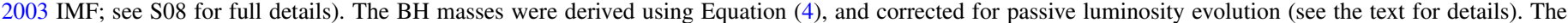

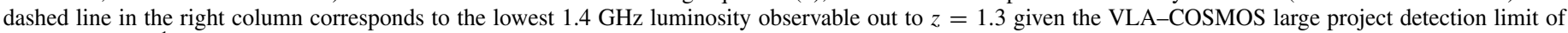
$\sim 50 \mu \mathrm{Jy}^{\text {beam }^{-1}}$.

evolution (see Hopkins et al. 2006b and references therein) we change the normalization constant of the above relation as a function of redshift following the results from Hopkins et al. (2006b, see short-dashed line in the bottom left panel in their Figure 2). In Figure 7 (bottom panels) we plot the estimated $\mathrm{BH}$ masses as a function of both redshift and radio $1.4 \mathrm{GHz}$ luminosity. The median $\mathrm{BH}$ mass of our radio $\mathrm{AGN}$ is $\log _{10} M_{\mathrm{BH}}=8.8$ with a standard deviation of 0.4 . Note that the apparent trend of $\mathrm{BH}$ mass with radio luminosity (bottom right panel in Figure 7) reflects the dependence of radio luminosity on redshift in our radio flux limited sample (see Figure 16 in S08) rather than a real trend.

The $\mathrm{BH}$ masses of our intermediate redshift radio AGN are comparable to the highest $\mathrm{BH}$ masses known in the local universe (see, e.g., McLure \& Dunlop 2002, 2004). This implies that the low-power radio AGN have assembled their $\mathrm{BH}$ masses already by these intermediate redshifts, and that their BHs cannot grow significantly since $z \sim 1.3$. Therefore, they must be in a mode of modest growth in $\mathrm{BH}$ mass. This result is in agreement with numerous findings in the literature implying that LERAGN accrete radiatively inefficiently, at sub-Eddington rates (Allen et al. 2006; Evans et al. 2006; Hardcastle et al. 2006, 2007).

Given all of the above, the composition of our VLACOSMOS AGN, the majority of which are shown to be LERAGN, is consistent with the properties of low-radio-power AGN in the local universe: Our intermediate redshift AGN have already by $z \sim 1$ assembled both their stellar and BH masses, comparable to the high-mass end of the galaxies known today.

On the other hand, powerful radio galaxies at high redshifts tend to show strong emission lines in their optical spectra
(Rawlings et al. 1989; Baum \& Heckman 1989; Rawlings \& Saunders 1991; Willott et al. 1999, 2000), and are often associated with ongoing star formation (Archibald et al. 2001; Greve et al. 2006; Seymour et al. 2008) as suggested by their bluer rest-frame colors (compared to red-sequence galaxies). Again, this is consistent with local observations of such sources. In numerous studies in the literature (e.g., Barthel 1989; Haas et al. 2004) these objects have been shown to accrete radiatively efficiently at high ( $\sim$ Eddington) accretion rates. Thus these HERAGN present a mode of significant $\mathrm{BH}$ growth-unlike the low-radio-power LERAGN.

To summarize, the properties of local and intermediateredshift radio AGN, as shown above, suggest that radio activity is triggered in similar populations of objects independent of their redshift, i.e. cosmic time. This is in agreement with the finding that the rest-frame optical colors of radio-source host galaxies do not change with redshift (Barger et al. 2007; S08; Huynh et al. 2008). In addition, there is converging evidence that low-radiopower AGN reflect a modest-radiatively inefficient-mode of $\mathrm{BH}$ growth, while high-radio-power AGN are undergoing a phase of significant-radiatively efficient-BH growth.

\section{THE STAR FORMATION QUENCHING AND RADIO-AGN TRIGGERING RATES}

One of the aims of this work is to study the link between radio AGN activity and star formation from an observational perspective. Thus, in order to investigate whether the processes responsible for the build up of the red galaxy sequence and the triggering of radio AGN mode are related or not, in this section we study the stellar mass properties of the red parent (Sec- 


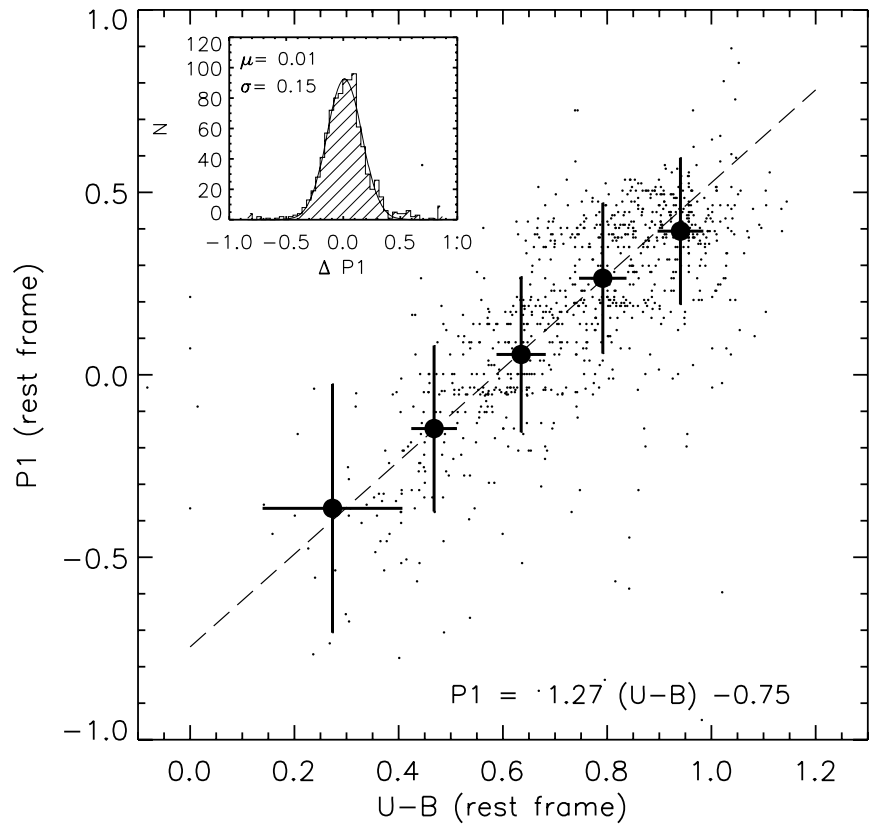

Figure 8. Comparison of the $\mathrm{P} 1$ and $U-B$ rest frame colors for 941 VLACOSMOS radio-optical sources (defined in S08; small dots). The dashed line shows the best linear fit (the analytic form is given in the bottom right of the panel), obtained using the median $(\mathrm{P} 1, U-B)$ values (large dots). The inset shows the dispersion of the fitted relation, where for a given galaxy $\Delta \mathrm{P} 1$ is the difference between its $\mathrm{P} 1$ value computed using its $U-B$ rest-frame color (obtained by Ilbert et al. 2009) and the one derived directly via SED fitting (as described in S08).

tion 6.1) and radio AGN samples (Section 6.2), and derive and compare the rates for the star formation quenching (Section 6.3) and (low radio power) AGN triggering (Section 6.4). We find no evidence that these two processes are related. This however does not exclude the possibility that the radio AGN phase may be responsible for preventing already assembled massive galaxies to grow higher in mass, as explored in Section 7.3.

\subsection{The Parent Galaxy Sample}

In order to derive the rates for star formation quenching and radio AGN triggering we need to define a control-parent galaxy sample of our VLA-COSMOS radio AGN. We define this sample using the COSMOS photometric redshift catalog (Ilbert et al. 2009) from which we select galaxies with the same optical magnitude, redshift and color criteria applied to our VLA-COSMOS AGN (see Section 2). The rest-frame optical color P1 (constrained from the $3200 \AA-5800 \AA$ range), which was used to select our VLA-COSMOS AGN is not available for the galaxies in the COSMOS photometric redshift catalog. Nonetheless, as the NUV-NIR galaxy SED has been shown to be a one-parameter family (e.g., Obrić et al. 2006; Smolčić et al. 2006), we can safely utilize another rest-frame color for the selection of the parent sample. In Figure 8 we show the correlation between $U-B$ and P1 rest-frame colors for all VLACOSMOS galaxies $(z \leqslant 1.3)$, and derive an analytic relation correlating $\mathrm{P} 1$ and $U-B$ (indicated in Figure 8 ). The criterion of P1 > 0.15, used to select the VLA-COSMOS AGN sample, corresponds to $U-B>0.71$. Thus we select the red galaxy parent sample by requiring $I_{\text {auto }} \leqslant 24,0.1<z_{\text {phot }} \leqslant 1.3$, and $U-$ $B>0.71$. The $U-B$ distribution for all (red and blue) galaxies, that satisfy the magnitude and redshift criteria, is shown in Figure 9. Note that the adopted $U-B$ threshold, based on the comparison with the $P 1$ values, corresponds almost exactly to

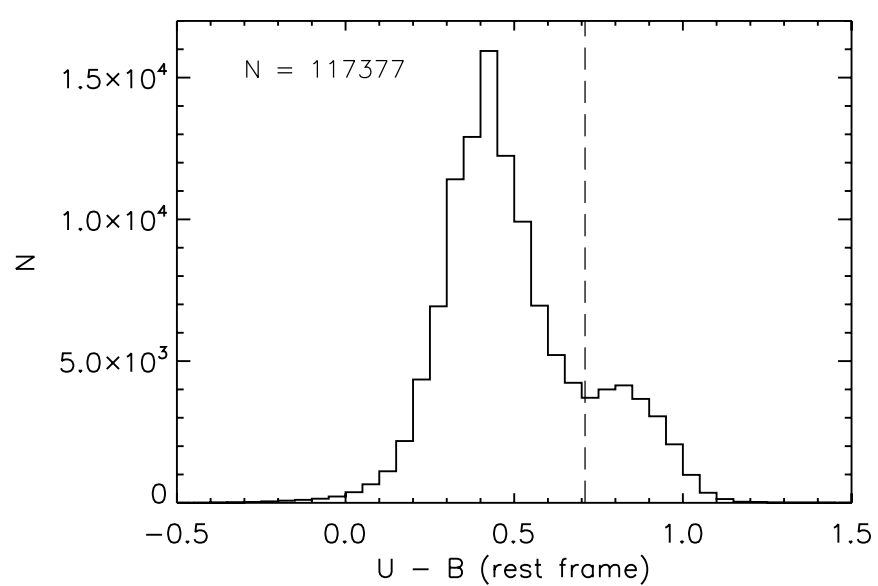

Figure 9. $U-B$ rest-frame color distribution for 117,377 galaxies $\left(I_{\text {auto }} \leqslant 24\right.$; $0.1<z_{\text {phot }} \leqslant 1.3$ ) drawn from the COSMOS photometric redshift catalog. The red-galaxy parent sample of our VLA-COSMOS radio AGN is selected by applying $U-B>0.71$ (dashed line) to this distribution (this is equivalent to $\mathrm{P} 1>0.15$ that was used to select our radio AGN; see Figure 8).

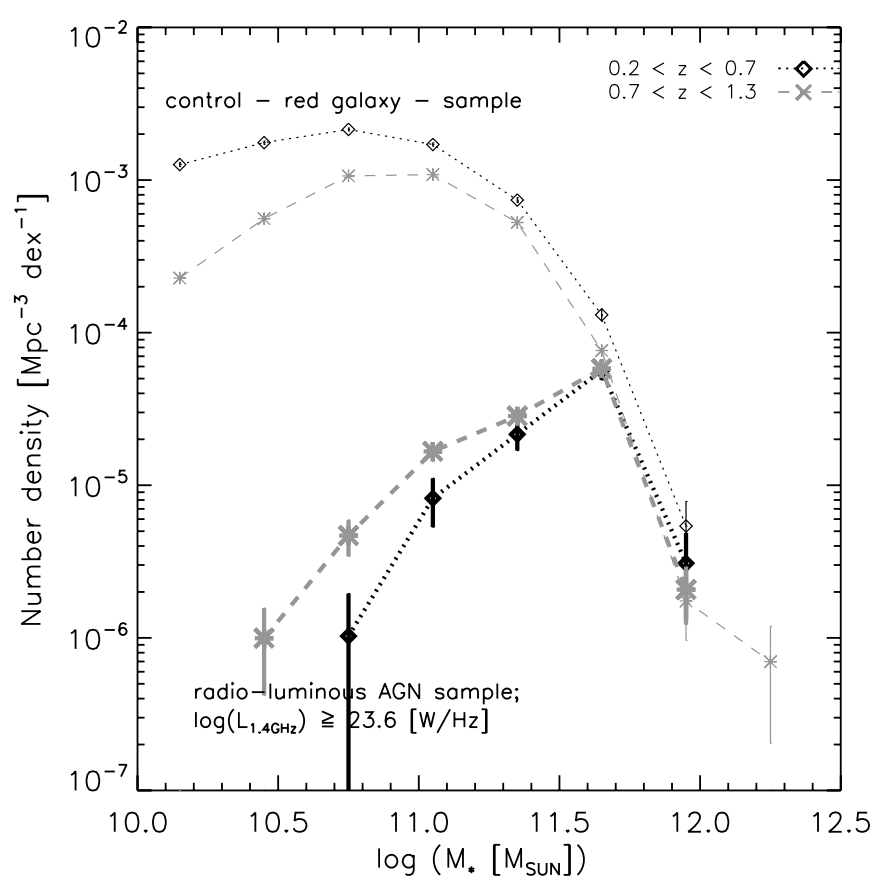

Figure 10. Stellar mass function, derived using the $1 / V_{\max }$ method, of the VLA-COSMOS radio AGN (volume limited, $L_{1.4 \mathrm{GHz}} \gtrsim 4 \times 10^{23} \mathrm{~W} \mathrm{~Hz}{ }^{-1}$; bold symbols) in two redshift ranges, indicated in the panel. For comparison, the stellar mass function of the red, parent galaxy sample in the same redshift ranges is also shown.

the $U-B$ value which separates the red from the blue galaxies. Our selection yields 21,525 galaxies in the red galaxy controlparent sample.

\subsection{The Stellar Mass Properties of VLA-COSMOS AGN}

Using the $1 / V_{\max }$ method we derive the stellar mass function (SMF) in two redshift bins $(0.2 \leqslant z \leqslant 0.7$ and $0.7<z \leqslant 1.3)$ for both our VLA-COSMOS AGN $\left(L_{1.4 \mathrm{GHz}} \gtrsim 4 \times 10^{23} \mathrm{~W}\right.$ $\mathrm{Hz}^{-1}$ which roughly corresponds to a radio volume limited sample), and the red parent galaxy sample. The stellar mass functions are shown in Figure 10. The SMF for the red galaxy sample agrees well with the recent results based on the GOODS- 


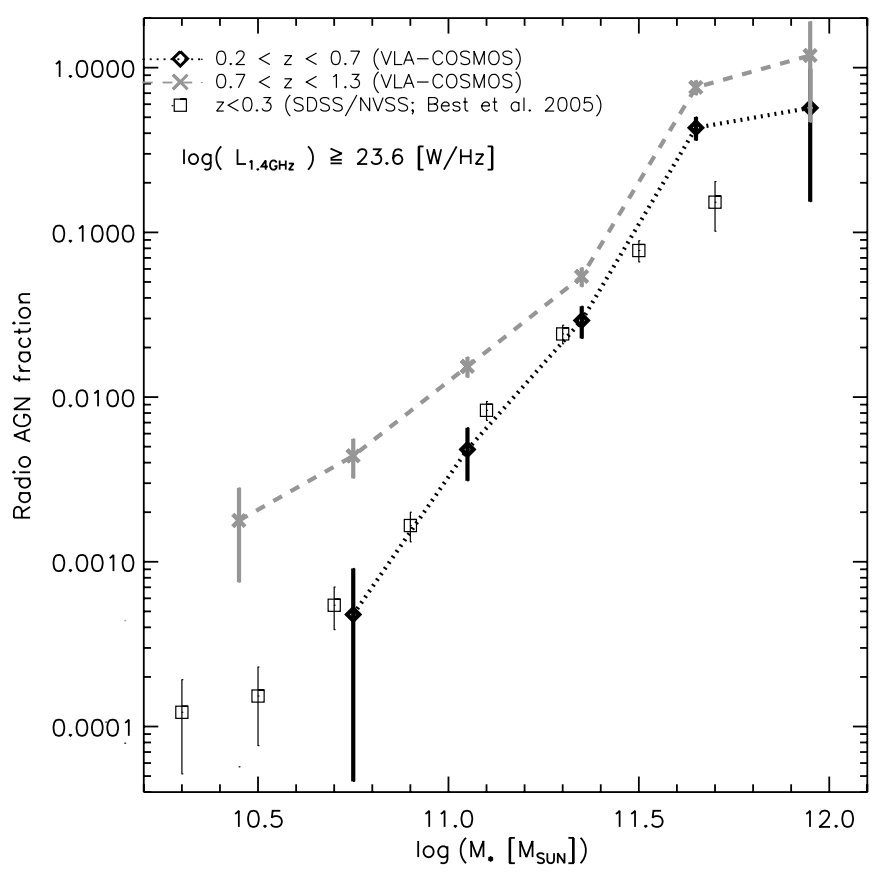

Figure 11. Fraction of radio luminous, VLA-COSMOS, AGN $\left(L_{1.4 \mathrm{GHz}} \gtrsim\right.$ $4 \times 10^{23} \mathrm{~W} \mathrm{~Hz}^{-1}$ ) as a function of stellar mass in two redshift ranges (bold symbols). Also shown is the radio AGN fraction based on the local $(z<0.3)$ SDSS/NVSS samples with the same radio-power limit imposed (Best et al. 2005). Note that no evolution of the radio AGN fraction is seen out to $z=0.7$.

MUSIC galaxy sample (Fontana et al. 2006). Similarly to what has been shown in the local universe for radio-luminous AGN (Best et al. 2005), at intermediate redshifts the SMFs of our radio AGN sample are strongly biased toward high stellar masses. Interestingly, the redshift evolution of the SMFs of the parent galaxy and radio AGN samples is reversed. While for a given stellar mass the comoving number density of red galaxies decreases with redshift, the number density of radioluminous AGNs increases. Our results are in good agreement with those of Tasse et al. (2008) based on radio observations of the XMM-LSS field.

We compute the fraction of radio-luminous AGNs as the ratio of the above derived mass functions in a given stellar mass bin. In Figure 11 we show the radio AGN fraction as a function of stellar mass in the two adopted redshift ranges, and compare it to the results based on the local SDSS and NVSS surveys (Best et al. 2005). The radio AGN fraction in our lower redshift $(0.2<z<0.7)$ bin agrees remarkably well with the local findings implying insignificant or absent evolution of the radio AGN fraction out to $z \sim 0.7$ at all stellar masses. However, there is a significant change in the fraction of radio-luminous AGN at higher redshifts $(0.7<z<1.3)$, in particular for host galaxies with stellar masses lower than $\log M_{*} \sim 11.3$. We investigate this further in Section 6.4.

\subsection{The Star Formation Quenching Rate}

Following Bundy et al. (2008), we define the star formation quenching rate, $\dot{Q}$, as the fraction of all galaxies in a given stellar mass bin that migrate to the red sequence per Gyr. This migration can occur through processes such as mass buildup (via "wet" mergers) or gas consumption (fading of an already massive galaxy to red colors). In Figure 12 (left panel) we plot the fraction of red galaxies (as defined in Section 6.1) relative to the number of all galaxies (see Figure 9) as a function of cosmic time for various stellar mass bins. The red galaxy fractions have been derived in the same way as the radio AGN fraction (see Section 6.2), but using the red and total galaxy samples. The slope ( $\alpha$; i.e., the change in fraction per Gyr) of this red-galaxysequence buildup with cosmic time, shown in the left panel of Figure 12, is then equivalent to the star formation quenching rate. We find that as stellar mass increases from $\log M_{*}$ of 10.6 to $11.7 M_{\odot} \dot{Q}$ decreases from $6 \% \mathrm{Gyr}^{-1}$ to $0.6 \% \mathrm{Gyr}^{-1}$. Note also that for the highest stellar masses the red sequence is already almost fully in place by $z \sim 1$.

\subsection{The Triggering Rate of Low-Radio-Power AGN}

In Section 6.3, we have derived the star formation quenching rate based on the buildup of the red galaxy population as a function of cosmic age (left panel in Figure 12). In the right panel in Figure 12 we now show the radio volume limited $\left(\log L_{1.4 \mathrm{GHz}} \geqslant 23.6\right)$ fraction of our VLA-COSMOS AGN relative to the abundance of the red parent galaxies as a function of cosmic time, for the various stellar mass ranges as shown in Figure 11. Contrary to the red galaxy fractions, our results yield that the radio AGN fraction decreases with cosmic age. At all stellar masses $\left(10.6<\log M_{*}<11.7\right)$ a larger fraction of radio-luminous AGN is present at earlier, compared to later, cosmic times. Furthermore, the fraction of radio-luminous AGN decreases more steeply with cosmic time for higher stellar masses. For example, at $\log M_{*} \sim 11.65$ the rate of decrease is $10.8 \% \mathrm{Gyr}^{-1}$, while at $\log M_{*} \sim 10.75$ it is only $0.1 \% \mathrm{Gyr}^{-1}$.

Given the fraction of radio AGN at a given cosmic time, we can estimate the average time that each single massive galaxy spends as a radio AGN, $\tau_{\text {radio }}$. Assuming that massive galaxies, that form the parent sample of our radio $A G N$, form around $z \sim 2.5-3$ and survive to $z \sim 0$, their lifetime is then $\sim 10 \mathrm{Gyr}$. Multiplying this lifetime by the fraction of observed radio AGN then yields an approximate estimate of $\tau_{\text {radio. At a given cosmic }}$ time, $\tau_{\text {radio }}$, which is in the range of roughly $10 \mathrm{Myr}-5 \mathrm{Gyr}$ for our VLA-COSMOS AGN (see the right panel in Figure 12), is thus related either to the average duration of a single radioepisode or to the frequency of radio-mode retriggering.

Independent calculations of radio-source lifetimes, $t_{\text {radio }}$, result in lifetimes of a few times $0.001 \mathrm{Gyr}$ to a few times $0.1 \mathrm{Gyr}$ (Alexander \& Leahy 1987; Shabala et al. 2008). Assuming that the radio-source lifetime does not significantly change with cosmic time, it is most likely that the computed $\tau_{\text {radio }}$ reflects the occurrence of multiple radio phases of a single massive galaxy at a certain cosmic time. A radio "on" phase of $0.001-0.1 \mathrm{Gyr}$, combined with the derived $\tau_{\text {radio }}$, implies then that radio AGN activity has been triggered more often in the past than is today, and that at a given cosmic time it is more often triggered in massive galaxies compared to lower-mass galaxies.

The minimum radio-AGN-triggering rate can be obtained by assuming the maximum $t_{\text {radio }}$ of $0.1 \mathrm{Gyr}$, and taking into account the above derived $\tau_{\text {radio }}(0.010-5 \mathrm{Gyr})$. It is then $0.1 \% \mathrm{Gyr}^{-1}$ $-50 \% \mathrm{Gyr}^{-1}$ for the lowest and highest stellar mass bins, respectively. Yet, in the previous section, we have computed a star formation quenching rate of $6 \% \mathrm{Gyr}^{-1}$ for the lowest and $0.6 \% \mathrm{Gyr}^{-1}$ for the highest stellar mass bins (see left panel in Figure 12). Hence, comparing these estimates yields that our derived radio AGN triggering rates are by many factors off the derived star formation quenching rates, especially for the highest stellar masses.

If the quenching of star formation, that causes the buildup of the red galaxy sequence, is related to a single episode 

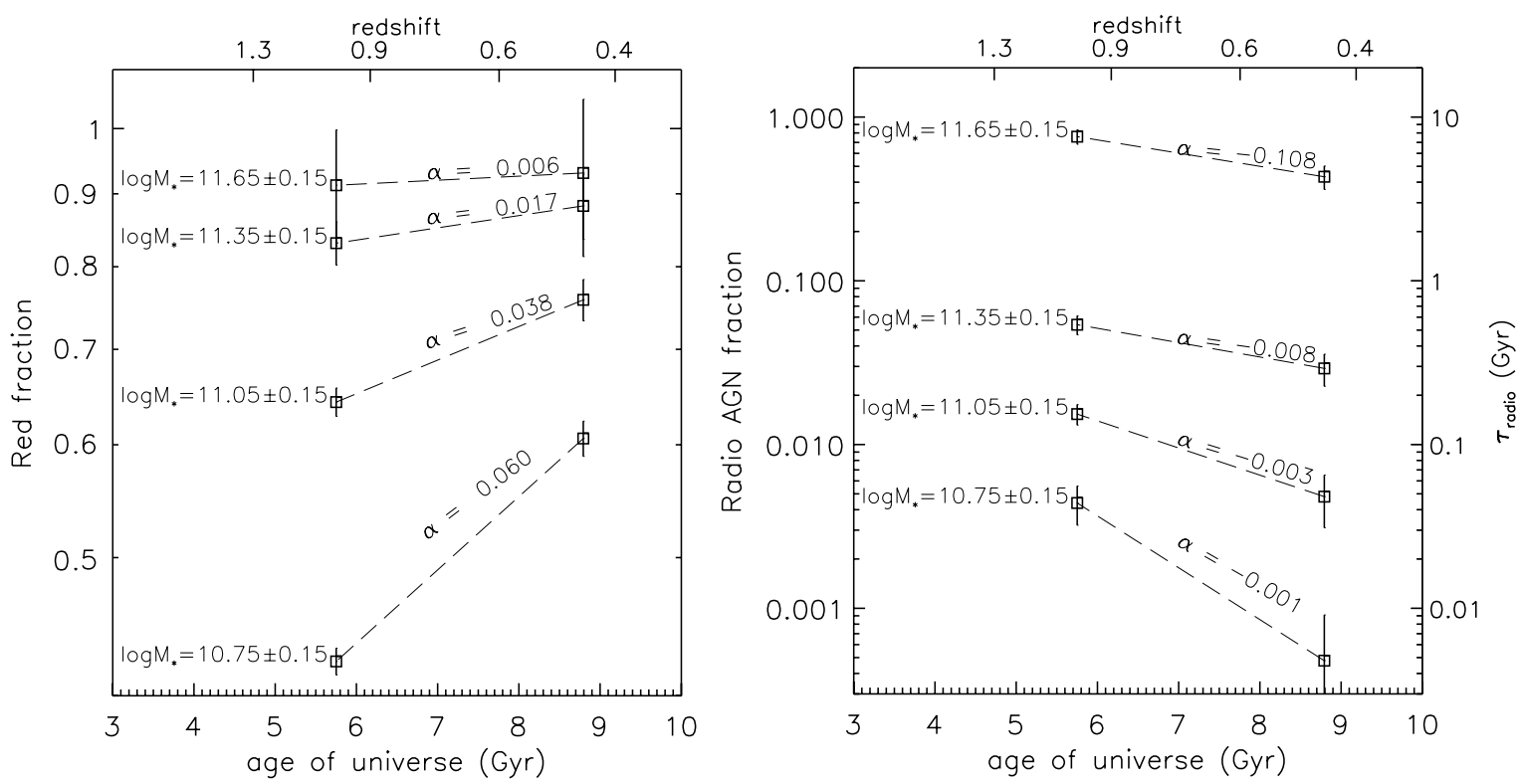

Figure 12. Left panel: the fraction of red galaxies $(P 1>0.15$; equivalent to $U-B>0.71$; left panel), compared to all galaxies, as a function of cosmic age for different stellar mass ranges (indicated in the panel). The right panel shows the fraction of radio AGN in the red, parent galaxy sample (as defined in the text) as a function of cosmic age for the various stellar mass bins. The fractional change with cosmic time has been characterized with its slope $(\alpha$; i.e., the change in fraction per Gyr), which is indicated for each stellar mass bin. The radio AGN fraction can be related to the average time a galaxy spends as a radio AGN ( $\tau_{\text {radio }}$; see the text for details), shown on the right-hand $y$-axis in the right panel. Note the different $y$ scales in the left and right panels. Note also that there is no correspondence between the increase of the red galaxy fraction, and the decrease of the radio AGN fraction with cosmic time.

(i.e., once the star formation is suppressed it does not restart at a significant level), then it is highly unlikely that a direct connection between the two phenomena-the low-radio-power AGN triggering and the star formation quenching rates-exists (see also, e.g., Bundy et al. 2008; Schawinski et al. 2009). However, these results do not exclude the possibility that lowradio-power AGN are responsible for preventing the galaxies to grow higher in mass, once they have been established as the most massive "red-and-dead" galaxies, as has been proposed in numerous cosmological models (Croton et al. 2006; Bower et al. 2006; Sijacki \& Springel 2006; Sijacki et al. 2007). This will be further explored in Section 7.3.

\section{IMPLICATIONS FOR GALAXY FORMATION}

\subsection{High-and Low-Radio-Power AGN: Different Stages of Galaxy Evolution}

In the last decades studies at various wavelength regimes have converged towards a widely accepted galaxy formation picture. Galaxies are thought to evolve in time from an initial stage with spiral morphology and blue optical colors towards elliptical morphologies with red optical colors (Bell et al. 2004a, 2004b; Borch et al. 2006; Faber et al. 2007; Brown et al. 2007; Hopkins et al. 2007). This evolution is not linear but happens through interspersed episodes of massive mass accretion onto the stellar body as well as their central massive BHs seen as quasars (Sanders \& Mirabel 1996; Sanders 2003). The blue-to-red galaxy evolution is accompanied with significant buildup of stellar and BH mass; the reddest galaxies observed in the universe are also the oldest and the most massive ones $\left(M_{*} \gtrsim 10^{11} M_{\odot}\right.$, e.g., Baldry et al. 2004, 2006; Faber et al. 2007). Mergers between galaxies, rich in cold gas ("wet" mergers), appear to be one of the key processes governing this mass buildup and the blue-to-red galaxy transformation (Sanders 2003; Bell et al. 2004a, 2004b; Borch et al. 2006; Faber et al. 2007; Brown et al. 2007). Furthermore, mergers are considered to be the major drivers of the growth of supermassive BHs in the centers of galaxies (Kauffmann \& Haehnelt 2000; Croton et al. 2006) as they enable large amounts of gas to be funneled to, and accrete onto the central $\mathrm{BH}$.

In the context of the general picture of galaxy evolution, the following scenario for the evolution of a radio AGN seems very plausible. High-radio-power AGNs are preferably found in galaxies that have not yet reached the highest stellar masses and show a strong decline with time of their number density. On the other hand, the hosts of low-power radio AGNs are generally old massive "red-and-dead" galaxies and have an almost constant space density. This implies that the radio triggering mechanism in a galaxy is a strong function of the host galaxy's properties, and it is likely that it is linked to different stages during the formation of massive old galaxies. In this scenario, the occurrence of the two radio modes would be naturally linked to the overall galaxy formation process, and their impact and timescales could be observationally constrained.

In particular, the HERAGN phase seems to be closely tied to major merger remnants (as many hosts are found to be disturbed, have blue colors and show signs of ongoing star formation, e.g., Heckman et al. 1986; Baum et al. 1992), as well as significant $\mathrm{BH}$ growth (supported by high $\sim$ Eddington accretion rates observed; see Section 5). These major mergers are critical for the formation of massive ellipticals. On the other hand, the LERAGN phase is mainly observed in the most massive galaxies, where a mechanism is required (Croton et al. 2006; Bower et al. 2006) which prevents intracluster gas from cooling onto the massive host galaxy. The properties of LERAGN such as lower amounts of gas and dust compared to HERAGN (de Koff et al. 1996; Leon et al. 2001; Müller et al. 2004; Evans et al. 2006; Emonts et al. 2007), a modest BH growth (Evans et al. 2006; Hopkins et al. 2006a; Cao 2007; Hardcastle et al. 2007), and fully built up stellar and BH masses already at $z \sim 1$ (Section 5, Kauffmann et al. 2008), support this interpretation.

In the context of the generally adopted red galaxy formation scenario, our results thus suggest that HERAGN (i.e., highradio-power AGN; $L_{1.4 \mathrm{GHz}} \gtrsim 10^{25} \mathrm{~W} \mathrm{~Hz}^{-1}$ ) occur in a stage 
of galaxy evolution presumably after a gas-rich merger which induces a phase of significant $\mathrm{BH}$ growth. This active radio phase may last $10^{6}-10^{7}$ yr (e.g., Alexander \& Leahy 1987; Shabala et al. 2008). Within the post-merging process the accumulated cold gas will be consumed by the host galaxy via star formation and/or BH accretion. LERAGN (i.e., low-radio-power AGNs; $L_{1.4 \mathrm{GHz}} \lesssim 10^{25} \mathrm{~W} \mathrm{~Hz}^{-1}$ ) then represent a later stage of the galaxy formation process, when the amount of cold gas in the host has already been reduced. In this phase, the galaxy's BH is accreting quiescently in a radiatively inefficient way, i.e., below a certain critical accretion rate in Eddington units, and most of the released energy is in kinetic form (see e.g., Hardcastle et al. 2007; Merloni \& Heinz 2008). This radio phase lasts a few times $10^{7}$ yr to a few times $10^{8} \mathrm{yr}$ (e.g., McNamara et al. 2005; Nulsen et al. 2005). Thus, our results imply that high-radiopower AGNs appear in an early stage, while low radio power occur in a late stage in the evolution of massive galaxies.

The evolution of the integrated luminosity density, shown in Figure 4, can be well explained in our scenario. This diagram can be interpreted as the evolution of $\Omega_{1.4 \mathrm{GHz}}$ for two radio luminous, but fundamentally different, types of galaxies where the HERAGN and LERAGN reflect modes of vigorous and modest $\mathrm{BH}$ growth, respectively.

The luminosity density of the HERAGN equivalent to a mode of vigorous $\mathrm{BH}$ growth evolves dramatically since $z=1.3$, consistent with e.g., the evolution of optically and X-rayselected quasars (see Willott et al. 2001; and also Boyle et al. 2000 and Ueda et al. 2003). The state of modest BH growth (in our case the LERAGN) evolves significantly slower, and it dominates the total comoving AGN radio power output at low redshifts $(z \lesssim 0.7)$. At these redshifts the most massive, "redand-dead", often central cluster galaxies associated with radio sources, are already in place (e.g., Blakeslee et al. 2003) thus providing an optimal environment for a self-regulating process of modest $\mathrm{BH}$ growth (at sub-Eddington accretion rates) and low-radio-power AGN activity.

In summary, the evolution of a radio-selected AGN population seems to be consistent with the possibly bimodal evolution of BH accretion modes (modest versus vigorous) that occur during different stages in the process of the formation of (massive) galaxies.

\subsection{The Co-evolution of Star Formation and Radio AGN Activity in Radio-Luminous Galaxies}

BH growth, especially merger-driven growth, is expected to be linked to starburst activity (Sanders \& Mirabel 1996; Boyle \& Terlevich 1998; Franceschini et al. 1999; Sanders 2003; Croton et al. 2006). Thus, in Figure 13, we compare the evolution of the integrated comoving $1.4 \mathrm{GHz}$ luminosity density for the VLACOSMOS star-forming galaxies (see S09 for details) and the total radio AGN population, likely reflecting two separate modes of $\mathrm{BH}$ accretion, as argued above. For the latter, $\Omega_{1.4 \mathrm{GHz}}$ has been obtained by co-adding the contributions of the low-power (VLA-COSMOS) and the high-radio-power AGNs (Willott et al. 2001; see Figure 4).

As the peak of the cosmic star formation and AGN activity occurs at $z>1$ (Hopkins 2004), we extrapolate $\Omega_{1.4 \mathrm{GHz}}$ to $z=2.5$ as follows. For the high-power AGN, we use the Willott et al. (2001) model which is well constrained by 3CRR, 6CE, and 7CRS data at $z<3$ (see Figure 1 in Willott et al. 2001). As can be seen from Figure 3, the full (weak plus powerful radio AGN) Willott et al. model describes well also the evolution of weak radio $\mathrm{AGN}$, at least at $z<1.3$. Here we assume that this

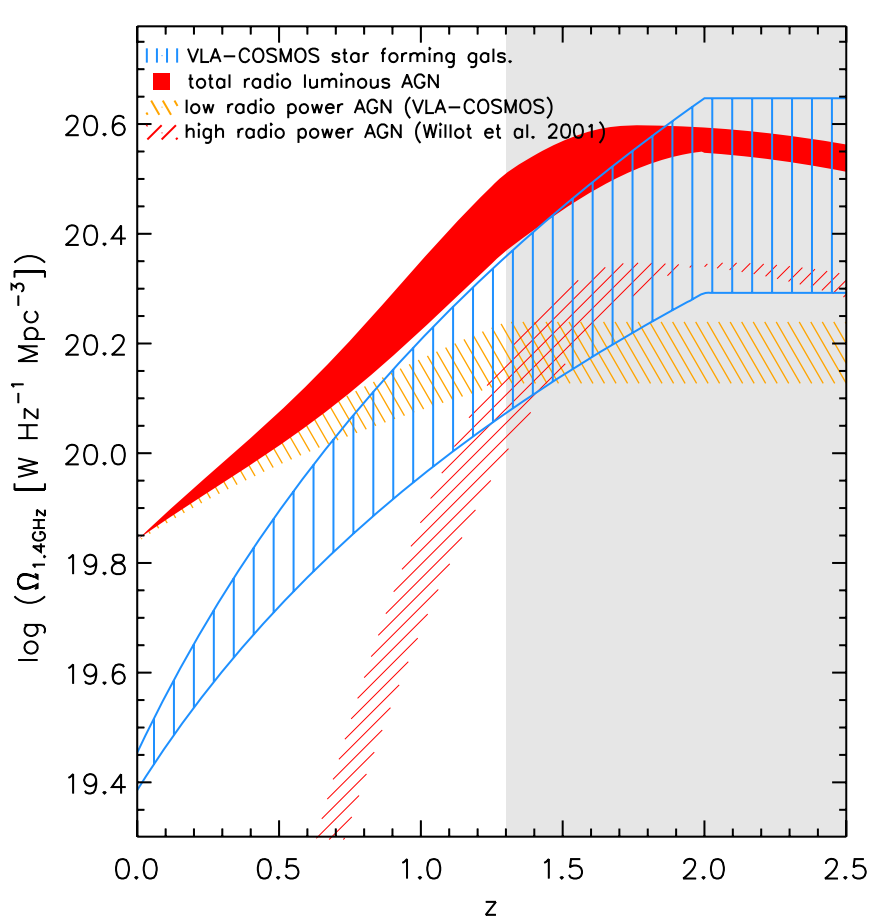

Figure 13. Evolution of the comoving $20 \mathrm{~cm}$ integrated luminosity density $\left(\Omega_{1.4 \mathrm{GHz}}\right)$ for all radio AGN (filled red curve) and star forming galaxies (vertically blue hatched curve) since $z=2.5$. The evolution of $\Omega_{1.4 \mathrm{GHz}}$ for the total AGN population is a superposition of the evolution of low (VLACOSMOS; orange hatched curve) and high- (model taken from Willott et al. 2001; red hatched region) radio-power AGN. The light-gray shaded area of the plot shows the redshift range where the evolution of the VLA-COSMOS AGN and star-forming galaxies has been extrapolated $(z>1.3$; see the text for details).

(A color version of this figure is available in the online journal.)

is the case also for $z>1.3$. Thus, in order to be consistent with this model, in which the low-power AGN population is assumed to stop evolving beyond a certain redshift, we assume that the comoving volume densities of VLA-COSMOS AGN remain constant for $z>1.3$. Note, however, that this may not necessarily be realistic if the comoving volume density of low-luminosity AGN exhibits a turnover, but at lower redshift compared to powerful radio sources (e.g., Waddington et al. 2001).

To extrapolate $\Omega_{1.4 \mathrm{GHz}}$ for the star-forming galaxies, we follow Hopkins (2004) who showed, based on a compilation of numerous studies based on independent data sets, that ceasing the evolution beyond $z=2$ (given the evolving radio-luminosity function for star-forming galaxies) reproduces the observed cosmic star formation history well (see, e.g., Figure 1 in Hopkins 2004). Thus, our extrapolation of $\Omega_{1.4 \mathrm{GHz}}$ beyond $z=1.3$ is fairly robust as it is based on independent results drawn from various data sets that constrain these cosmic times well.

The integrated comoving $20 \mathrm{~cm}$ luminosity density for radioluminous star-forming and AGN galaxies, shown in Figure 13, appears to evolve coevally at high redshifts $(0.7 \lesssim z<2.5)$, where powerful AGN significantly contribute to the $\Omega_{1.4 \mathrm{GHz}}$. Both seem to flatten in the redshift range of about 1.5-2. Although not conclusive, this is suggestive of a link between the process of star formation and radio AGN activity (see also, e.g., Boyle \& Terlevich 1998; Franceschini et al. 1999). This can be understood in the scenario of galaxy formation where gas-rich mergers of spiral galaxies govern the formation of gas-poor elliptical galaxies. As the galaxies merge, the bulk of their cold gas, that was originally distributed throughout 


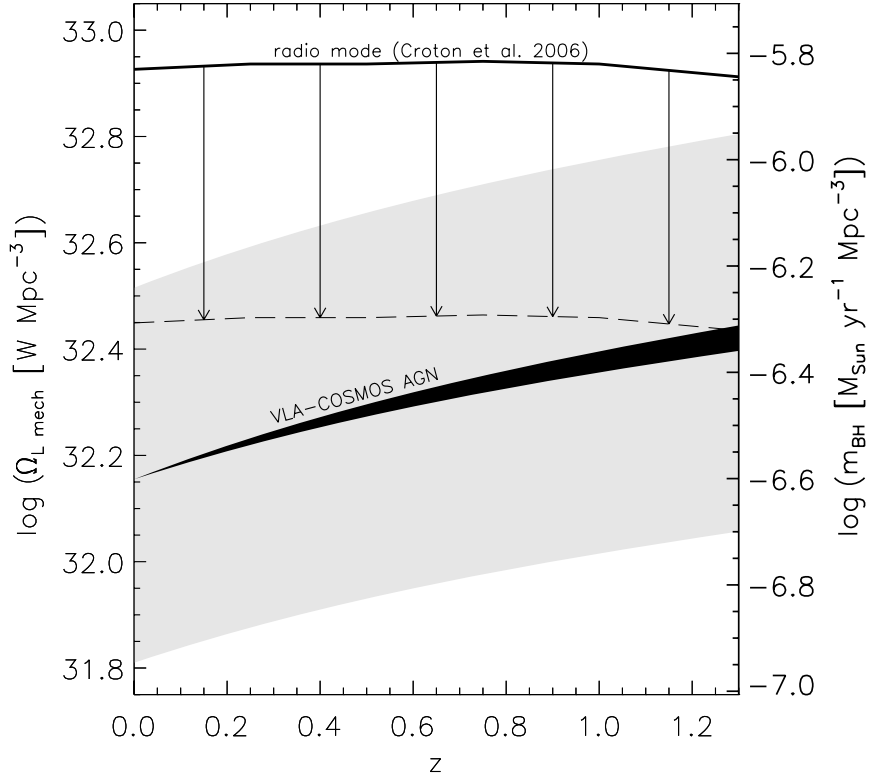

Figure 14. Cosmic evolution of the volume-averaged mechanical heating rate $\Omega_{\mathrm{L} \text { mech }}$ for low-power VLA-COSMOS AGN, which are likely candidates for the "radio mode" heating invoked in cosmological models (filled curve). The volume-averaged accretion rate is shown on the right-hand side $y$-axis (see the text for details). The uncertainties in the correlation between $L_{1.4 \mathrm{GHz}}$ and $L_{\text {mech }}$ (given in Bîrzan et al. 2008) are illustrated by the light-gray shaded area. Also shown is the evolution predicted in the Croton et al. (2006) semianalytic model (thick line), and its possible value lowered by a factor of 2-3 (indicated by arrows) due to a systematic overestimation of the heating rate in the model (see the text for details).

the disk of the merging constituents, is funneled into the inner few kpc of the merging system and fuels both star formation and AGN activity (possibly sequentially; Sanders 2003). Thus, coevolution of star-forming and powerful-radio AGN activities is predominantly expected in systems with significant amounts of cold gas, consistent with the properties found in HERAGN.

On the other hand, the evolution of the integrated comoving $20 \mathrm{~cm}$ luminosity density for star-forming and radio-AGN galaxies decouples below $z \sim 0.7$ (see Figure 13) where lowradio-power AGNs with modest $\mathrm{BH}$ growth dominate the total AGNs radio-power output in the universe (see Figure 4). In the context of our high-to-low-radio-power transition scenario proposed in the previous section, this AGN activity is triggered in a later evolutionary stage of a massive galaxy (corresponding to LERAGN), where a large fraction of the gas reservoir has already been consumed. Thus, a close link between star formation and AGN activity is not expected in this regime. Such an expectation is also supported by our results on the quenching of star formation in the context of the buildup of the red galaxy sequence, derived in Section 6.3. We have shown that processes causing the star formation quenching are not likely to be also governing the triggering of low-radio-power AGN phases. If they are responsible for the decline in the star formation rate density since $z \sim 1$ (at least at the high stellar mass end; see e.g., S09), then no connection between $\Omega_{1.4 \mathrm{GHz}}$ for low-power radio AGN and star-forming galaxies is expected.

We stress that the radio energy injection into the surrounding medium of such quiescent modes of $\mathrm{BH}$ accretion has been invoked in numerous cosmological models (e.g., Croton et al. 2006; Bower et al. 2006; Sijacki \& Springel 2006; Sijacki et al. 2007 ) as the main heating mechanism responsible for preventing further star formation in the given host galaxy (assuming that every massive red galaxy undergoes a phase of radio AGN activity). This process was the key ingredient that allowed a good reproduction of e.g., the high-mass end of the galaxy stellar mass function in the models. To shed light on the plausibility of such a process we explore in the following section the injection energies of our VLA-COSMOS AGN, and compare them to the recipes used in cosmological simulations.

\subsection{The Evolution of Radio AGN Feedback in Massive Galaxies}

In this section we derive the mechanical energy injected into the surrounding medium by low-power (VLA-COSMOS) radio AGN, which are possible candidates for preventing star formation in massive galaxies as proposed in recent cosmological models (Croton et al. 2006; Bower et al. 2006; Sijacki \& Springel 2006; Sijacki et al. 2007), and defined as 'radio mode' (see Croton et al. 2006 for details).

Monochromatic radio luminosity is not a direct indicator of the mechanical energy output of a radio galaxy into its surrounding medium. The latter can be estimated making use of the observed interplay in galaxy clusters between radio galaxies and the hot X-ray-emitting intracluster gas, where radio jets induce cavities in the hot gas and inflate buoyantly rising bubbles (see, e.g., Bîrzan et al. 2004, 2008; Allen et al. 2006). As the total energy within a bubble is the sum of its internal energy and the PdV work done by its inflation, the mechanical radio luminosity is given by the ratio of this total energy and the age of the bubble (see, e.g., Bîrzan et al. 2004, 2008; Allen et al. 2006). Using mechanical luminosities derived by Bîrzan et al. (2004) for a sample of 18 radio galaxies in clusters or groups, Best et al. (2006) have found that the relation of mechanical and monochromatic radio power is best described by a power law, however, with a large scatter (see Figure 1 and Equation (2) in Best et al. 2004). Based on this relation, they have computed the total comoving volume-averaged mechanical heating rate, $\Omega_{\mathrm{L} \text { mech }}$, in the local universe provided by radio-luminous AGN, which was found to be a factor of 10-20 lower than the local density of radio mode heating predicted in the Croton et al. (2006) semianalytic model.

Here, we follow the prescription of Best et al. (2006) to estimate the evolution of $\Omega_{\mathrm{L} \text { mech }}$, using the most recent calibration for the conversion of monochromatic radio to mechanical power (Bîrzan et al. 2008). First, at each redshift, we convert the radio AGN monochromatic luminosity function (given by our best fit PLE and PDE models; see Section 4.1) into a mechanical luminosity density function using Equation (16), given by Bîrzan et al. (2008). Then we integrate it above a minimum mechanical luminosity equivalent to $L_{1.4 \mathrm{GHz}}=10^{21} \mathrm{~W} \mathrm{~Hz}^{-1}$. The resulting evolution of $\Omega_{\mathrm{L} \text { mech }}$ is shown in Figure 14, where we compare it to the density of the radio mode heating given in the Croton et al. (2006) cosmological model. The latter has been obtained from Figure 3 in Croton et al., where we converted their comoving $\mathrm{BH}$ accretion rate density, $\dot{m}_{\mathrm{BH}}$ (shown on the right-hand side of the $y$-axis), into the volume-averaged heating rate utilizing $\Omega_{\mathrm{L} \text { mech }}=\eta \dot{m}_{\mathrm{BH}} c^{2}$, where $\eta$ is the standard efficiency of the conversion of mass into energy $(\eta=0.1)$, and $c$ is the speed of light (see also Equation (11) of Croton et al. 2006).

It is important to note that both the model- and observationbased heating rates are subject to large uncertainties. The modelcooling rates are likely to be overestimated by a factor of 2-3 (indicated in Figure 14) due to the nature of the underlying cooling flow model (Best et al. 2006). On the other hand, 
the observationally derived heating rates have uncertainties of the order of $\sim 0.8$ dex due to the scatter in the conversion of monochromatic to mechanical radio luminosities. Furthermore, they may be strongly underestimated (up to a factor of 6), as the work done during the inflation of a bubble may be significantly higher than the reversible pdV work usually assumed in the computation of mechanical luminosities (see Binney et al. 2007 for details). In addition, the minimum mechanical power equivalent to $L_{1.4 \mathrm{GHz}}=10^{21} \mathrm{~W} \mathrm{~Hz}^{-1}$ has been chosen rather arbitrarily. For example, a lower threshold would yield a higher $\Omega_{\mathrm{Lmech}}$. Nonetheless, the qualitative agreement between the model and observations seen in Figure 14 is encouraging for the idea that radio-luminous AGNs play an important role in the process of galaxy evolution.

Based on two samples of local massive red galaxies with available optical, radio and X-ray observations, Best et al. (2006) have shown that radio source activity may indeed control the growth rate of the host galaxy, as has been proposed in cosmological models. Best et al. have found that, across a wide range of stellar masses, the radio-source heating rate agrees remarkably well with the cooling rate of the host galaxy's surrounding hot-gas halo. Averaged over time, the recurring radio source activity can then balance the radiative losses from its surrounding hot-gas halo and thereby suppress the cooling of the gas, and in this way control the growth rate of the galaxy (see, e.g., Croton et al. 2006). Here we have shown that various properties of $z \sim 1$ (low power) radio AGNs are comparable to the properties of local radio AGNs. Thus it is very likely that the same radio heating - hot gas cooling interplay, observed locally, takes place also in $z \sim 1$ radio AGN. This then strongly argues in favor of the plausibility that (low-power) radio AGN activity (i.e., "radio mode") is a key factor that prevents further mass growth in massive "red-and-dead" galaxies across cosmic times, at least since $\sim 4.5 \mathrm{Gyr}$ after the Big Bang.

\section{SUMMARY AND CONCLUSIONS}

Using the largest sample of low-luminosity radio AGN at intermediate redshifts available to date drawn from the VLACOSMOS survey, we explored the evolution and composition of the radio AGN population out to a redshift of $z \sim 1$. In particular, we derived the radio-AGN luminosity function in several redshift bins. Compared to powerful $\left(L_{1.4 \mathrm{GHz}}>5 \times 10^{25} \mathrm{~W}\right.$ $\left.\mathrm{Hz}^{-1}\right)$ radio $\mathrm{AGN}$, the low-radio-power $\left(L_{1.4 \mathrm{GHz}} \lesssim 5 \times\right.$ $10^{25} \mathrm{~W} \mathrm{~Hz}^{-1}$ ) AGN probed here show only a weak decline in their luminosity density since $z=1.3$.

An analysis of the host properties of weak and powerful radio AGN shows that they form two relatively distinct classes, with powerful radio AGNs being related to modes of vigorous $\mathrm{BH}$ growth, whereas weak radio AGNs are related to only modest $\mathrm{BH}$ growth. This is also reflected in the types of host galaxies with weak radio AGN being preferentially found in the most massive and evolved galaxies (even out to $z \sim 1$ ).

Joint analysis of the evolution of the $20 \mathrm{~cm}$ luminosity density of the AGN and the star formation rate density suggests that the powerful radio sources are preferably triggered in major mergers following shortly after the phase of massive star formation at high redshifts. This would also naturally explain the strong decline of these sources below a redshift of $z=1$. As the volume density of weak radio AGN stays fairly constant, they can significantly contribute to the heating of the their surrounding medium and thus inhibit gas accretion onto their host galaxies as recently suggested for the radio mode in cosmological models.
The authors are grateful to P. N. Best for especially deriving the SDSS/NVSS radio source fractions as a function of stellar mass with a radio luminosity cut that matches the VLACOSMOS survey data. C.C. acknowledges support from the Max-Planck Society and the Alexander von Humboldt Foundation through the Max-Planck-Forschungspreis 2005. G.Z. and S.B. acknowledge support from an INAF contract PRIN2006/1.06.10.08 and an ASI grant ASI/COFIS I/026/07/0. TP acknowledges support from PSC-CUNY grant no. 69612-00 38.

\section{REFERENCES}

Alexander, P., \& Leahy, J. P. 1987, MNRAS, 225, 1

Allen, S. W., Dunn, R. J. H., Fabian, A. C., Taylor, G. B., \& Reynolds, C. S. 2006, MNRAS, 372, 21

Archibald, E. N., Dunlop, J. S., Hughes, D. H., Rawlings, S., Eales, S. A., \& Ivison, R. J. 2001, MNRAS, 323, 417

Baldi, R. D., \& Capetti, A. 2008, A\&A, 489, 989

Baldry, I. K., Glazebrook, K., Brinkmann, J., Ivezić, Ž., Lupton, R. H., Nichol, R. C., \& Szalay, A. S. 2004, ApJ, 600, 681

Baldry, I. K., Balogh, M. L., Bower, R. G., Glazebrook, K., Nichol, R. C., Bamford, S. P., \& Budavari, T. 2006, MNRAS, 373, 469

Baldwin, J. A., Phillips, M. M., \& Terlevich, R. 1981, PASP, 93, 5

Barger, A. J., Cowie, L. L., \& Wang, W.-H. 2007, ApJ, 654, 764

Barthel, P. D. 1989, ApJ, 336, 606

Baum, S. A., Heckman, T. M., \& van Breugel, W. 1992, ApJ, 389, 208

Baum, S. A, \& Heckman, T. 1989, ApJ, 336, 702

Bell, E. F., et al. 2004a, ApJ, 608, 752

Bell, E. F., et al. 2004b, ApJ, 600, L11

Best, P. N. 2004, MNRAS, 351, 70

Best, P. N., Kaiser, C. R., Heckman, T. M., \& Kauffmann, G. 2006, MNRAS, 368, L67

Best, P. N., et al. 2005, MNRAS, 362, 9

Best, P. N. 2004, MNARS, 351, 70

Binney, J., Alouani Bibi, F., \& Omma, H. 2007, MNRAS, 377, 142

Bîrzan, L., McNamara, B. R., Nulsen, P. E. J., Carilli, C. L., \& Wise, M. W. 2008, ApJ, 686, 859

Bîrzan, L., Rafferty, D. A., McNamara, B. R., Wise, M. W., \& Nulsen, P. E. J. 2004, ApJ, 607, 800

Blakeslee, J. P., et al. 2003, ApJ, 596, L143

Bondi, M., Ciliegi, P., Schinnerer, E., Smolčić, V., Jahnke, K., Carilli, C., \& Zamorani, G. 2008, ApJ, 681, 1129

Borch, A., et al. 2006, A\&A, 453, 869

Bower, R. G., Benson, A. J., Malbon, R., Helly, J. C., Frenk, C. S., Baugh, C. M., Cole, S., \& Lacey, C. G. 2006, MNRAS, 370, 645

Boyle, B. J., Shanks, T., Croom, S. M., Smith, R. J., Miller, L., Loaring, N., \& Heymans, C. 2000, MNRAS, 317, 1014

Boyle, B. J., \& Terlevich, R. J. 1998, MNRAS, 293, L49

Brown, M. J. I., Dey, A., Jannuzi, B. T., Brand, K., Benson, A. J., Brodwin, M., Croton, D. J., \& Eisenhardt, P. R. 2007, ApJ, 654, 858

Brusa, M., et al. 2009, ApJ, 693, 8

Bruzual, G., \& Charlot, S. 2003, MNRAS, 344, 1000

Bundy, K., et al. 2008, ApJ, 681, 931

Cao, X. 2007, ApJ, 659, 950

Capak, E., et al. 2007, ApJS, 172, 99

Chabrier, G. 2003, PASP, 115, 763

Chiaberge, M., Capetti, A., \& Macchetto, F. D. 2005, ApJ, 625, 716

Clewley, L., \& Jarvis, M. J. 2004, MNRAS, 352, 909

Condon, J. J., Cotton, W. D., \& Broderick, J. J. 2002, AJ, 124, 675

Cowie, L. L., Barger, A. J., Fomalont, E. B., \& Capak, P. 2004, ApJ, 603, L69

Croton, D. J., et al. 2006, MNRAS, 365, 11

Daddi, E., et al. 2007, ApJ, 670, 173

de Koff, S., Baum, S. A., Sparks, W. B., Biretta, J., Golombek, D., Macchetto, F., McCarthy, P., \& Miley, G. K. 1996, ApJS, 107, 621

de Koff, S., et al. 2000, ApJS, 129, 33

Donoso, E., Best, P. N., \& Kauffmann, G. 2008, MNRAS, 392, 617

Dunlop, J. S., \& Peacock, J. A. 1990, MNRAS, 247, 19

Emonts, B. H. C., Morganti, R., Oosterloo, T. A., van der Hulst, J. M., van Moorsel, G., \& Tadhunter, C. N. 2007, A\&A, 464, L1

Evans, A. S., Mazzarella, J. M., Surace, J. A., Frayer, D. T., Iwasawa, K., \& Sanders, D. B. 2005, ApJS, 159, 197

Evans, D. A., Worrall, D. M., Hardcastle, M. J., Kraft, R. P., \& Birkinshaw, M. 2006, ApJ, 642, 96 
Faber, S. M., et al. 2007, ApJ, 665, 265

Fabian, A. C. 1994, ARA\&A, 32, 277

Fabian, A. C., et al. 2003, MNRAS, 344, L43

Fanaroff, B. L., \& Riley, J. M. 1974, MNRAS, 167, 31

Fontana, A., et al. 2006, A\&A, 459, 745

Forman, W., et al. 2005, ApJ, 635, 894

Franceschini, A., Hasinger, G., Miyaji, T., \& Malquori, D. 1999, MNRAS, 310 , L5

Garham, A. W. 2007, MNRAS, 379, 711

Greve, T. R., Ivison, R. J., \& Stevens, J. A. 2006, Astronomische Nachrichten, 327,208

Haas, M., et al. 2004, A\&A, 424, 531

Hardcastle, M. J., Evans, D. A., \& Croston, J. H. 2006, MNRAS, 370, 1893

Hardcastle, M. J., Evans, D. A., \& Croston, J. H. 2007, MNRAS, 376, 1849

Heckman, T. M., Smith, E. P., Baum, S. A., van Breugel, W. J. M., Miley, G. K., Illingworth, G. D., Bothun, G. D., \& Balick, B. 1986, ApJ, 311, 526

Hine, R. G., \& Longair, M. S. 1979, MNRAS, 188, 111

Ho, L. C. 2005, Ap\&SS, 300, 219

Hopkins, A. M. 2004, ApJ, 615, 209

Hopkins, P. F., Bundy, K., Hernquist, L., \& Ellis, R. S. 2007, ApJ, 659, 976

Hopkins, P. F., Narayan, R., \& Hernquist, L. 2006, ApJ, 643, 641

Hopkins, P. F., Robertson, B., Krause, E., Hernquist, L., \& Cox, T. J. 2006, ApJ, 652, 107

Huynh, M. T., et al. 2008, AJ, 135, 2470

Ilbert, O., et al. 2009, ApJ, 690, 1236

Kauffmann, G., \& Haehnelt, M. 2000, MNRAS, 311, 576

Kauffmann, G., Heckman, T. M., \& Best, P. N. 2008, MNRAS, 384, 953

Körding, E. G., Jester, S., \& Fender, R. 2008, MNRAS, 383, 277

Lacy, M., et al. 2004, ApJS, 154, 166

Laing, R. A., Jenkins, C. R., Wall, J. V., \& Unger, S. W. 1994, in ASP Conf Ser. 54, The Physics of Active Galaxies, ed. G. V. Bicknell, M. A. Dopita, \& P. J. Quinn (San Francisco, CA: ASP), 201

Ledlow, M. J., \& Owen, F. N. 1996, AJ, 112, 9

Le Floc'h, E., et al. 2005, ApJ, 632, 169

Leon, S., Lim, J., Combes, F., \& van Trung, D. 2001, in QSO Hosts and Their Environments, Proceedings of an International Workshop, held 2001 January 10-12, in Granada, Spain, ed. I. Márquez, et al. (Dordrecht: Kluwer), 185

Marconi, A., \& Hunt, L. K. 2003, ApJ, 589, L21

Mauch, T., \& Sadler, E. M. 2007, MNRAS, 375, 931

McLure, R. J., \& Dunlop, J. S. 2002, MNRAS, 331, 795

McLure, R. J., \& Dunlop, J. S. 2004, MNRAS, 352, 1390

McNamara, B. R., Nulsen, P. E. J., Wise, M. W., Rafferty, D. A., Carilli, C., Sarazin, C. L., \& Blanton, E. L. 2005, Nature, 433, 45

Merloni, A., \& Heinz, S. 2008, MNRAS, 388, 1011
Müller, S. A. H., Haas, M., Siebenmorgen, R., Klaas, U., Meisenheimer, K., Chini, R., \& Albrecht, M. 2004, A\&A, 426, L29

Nulsen, P. E. J., McNamara, B. R., Wise, M. W., \& David, L. P. 2005, ApJ, 628 , 629

Obrić, M., et al. 2006, MNRAS, 370, 1677

Owen, F. N. 1993, in Jets in Extragalactic Radio Sources, Vol. 421, ed. H.-J. Röser \& K. Meisenheimer (Berlin: Springer), 273

Rawlings, S., \& Saunders, R. 1991, Nature, 349, 138

Rawlings, S., Saunders, R., Eales, S. A., \& Mackay, C. D. 1989, MNRAS, 240, 701

Sadler, E. M., et al. 2002, MNRAS, 329, 227

Sadler, E. M., et al. 2007, MNRAS, 381, 211

Sanders, D. B. 2003, J. Korean Astron. Soc., 36, 149

Sanders, D. B., \& Mirabel, I. F. 1996, ARA\&A, 34, 749

Schawinski, K., et al. 2009, ApJ, 690, 1672

Schinnerer, E., et al. 2007, ApJS, 172, 46

Schmidt, M. 1968, ApJ, 151, 393

Schmidt, M., Schneider, D. P., \& Gunn, J. E. 1995, AJ, 110, 68

Scoville, E., et al. 2007, ApJS, 172, 1

Sijacki, D., \& Springel, V. 2006, MNRAS, 366, 397

Seymour, N., et al. 2008, ApJ, 681, L1

Shabala, S. S., Ash, S., Alexander, P., \& Riley, J. M. 2008, MNRAS, 388, 625

Sijacki, D., Springel, V., di Matteo, T., \& Hernquist, L. 2007, MNRAS, 380, 877

Silverman, J. D., et al. 2008, ApJ, 679, 118

Smolčić, V., et al. 2006, MNRAS, 371, 121

Smolčić, V., et al. 2008, ApJS, 177, 14

Smolčić, V., et al. 2009, ApJ, 690, 610

Solomon, P. M., \& Vanden Bout, P. A. 2005, ARA\&A, 43, 677

Stern, D., et al. 2005, ApJ, 631, 163

Tasse, C., Best, P. N., Röttgering, H., \& Le Borgne, D. 2008, A\&A, 490, 893

Trump, J. R., et al. 2007, ApJS, 172, 383

Ueda, Y., Akiyama, M., Ohta, K., \& Miyaji, T. 2003, ApJ, 598, 886

Waddington, I., Dunlop, J. S., Peacock, J. A., \& Windhorst, R. A. 2001, MNRAS, 328,882

White, S. D. M., \& Frenk, C. S. 1991, ApJ, 379, 52

White, S. D. M., \& Rees, M. J. 1978, MNRAS, 183, 341

Willott, C. J., Rawlings, S., Blundell, K. M., \& Lacy, M. 1999, MNRAS, 309, 1017

Willott, C. J., Rawlings, S., Blundell, K. M., \& Lacy, M. 2000, MNRAS, 316, 449

Willott, C. J., Rawlings, S., Blundell, K. M., Lacy, M., \& Eales, S. A. 2001, MNRAS, 322, 536 